\title{
Автоволны. Самоорганизация
}

УДК 517.98.537

\section{КЛАССИЧЕСКИЕ ДВУМЕРНЫЕ МОДЕЛИ КУЧИ ПЕСКА}

\author{
А. В. Подлазов \\ Институт прикладной математики им. М.В. Келдыша РАН \\ 125047, Москва, Миусская пл., д.4 \\ E-mail: tiger-cub@mail.ru
}

Работа посвящена изучению моделей кучи песка - открытых нелинейных систем, демонстрирующих возникновение лавинообразного развивающегося отклика на единичное возмущение устойчивого состояния. Подробно рассмотрены пять наиболее известных вариантов правил в двумерной постановке - модели Дхара-Рамасвами, Пастор-СаторрасаВеспиньяни, Федеров, Манны и Бака-Танга-Визенфельда.

Для первых четырех моделей были известны аналитические решения, полученные различными способами, а для пятой - причины, препятствовавшие построению решения. Обобщение этих результатов позволяет выработать единый подход к теоретическому исследованию самоорганизованно-критических явлений.

Самоорганизация в критическое состояние приводит к возникновению масштабноинвариантных свойств, статистическое описание которых в общем случае не может быть дано на основе правил изучаемых моделей. Между локальным поведением их элементов на микроуровне и целостным поведением всей системы на макроуровне посредничают модели промежуточного уровня. Их правила не выводятся из правил исходных моделей, а формулируются на основе физической интуиции, результатов компьютерного эксперимента и общих представлений о динамических процессах, удерживающих систему вблизи критической точки. На промежуточном уровне коллективная динамика всех рассмотренных моделей сведена к привычным для математической физики процессам, в первую очередь - к несимметричному случайному блужданию. На этой основе предложены сходные методики решения моделей, причем модель БТВ решена впервые.

Для рассматриваемых моделей аналитически определены все критические индексы, на основе чего проанализировано влияние особенностей правил моделей на их общие свойства.

Важнейшей деталью правил является их стохастичность или детерминированность. Первая увеличивает количество характеристик лавины, различающихся по свойствам, а вторая помогает крупным лавинам помещаться в систему конечного размера и приводит к возникновению у системы как у целого динамических симметрий, отсутствующих на уровне правил поведения ее отдельных элементов.

Ключевые слова: Самоорганизованная критичность, модели кучи песка, масштабная инвариантность, степенные распределения, конечно-размерный скейлинг, модели промежуточного уровня, случайные блуждания, спонтанная анизотропия, динамические симметрии.

DOI: $10.18500 / 0869-6632-2016-24-4-39-70$

Ссылка на статью: Подлазов А.В. Классические двумерные модели кучи песка // Известия вузов. Прикладная нелинейная динамика. 2016. Т. 24, № 4. С. 39-70. 


\section{Введение}

Одним из ключевых признаков сложного поведения является масштабная инвариантность происходящих процессов, т.е. отсутствие у описывающих их величин собственных (не связанных с размерами системы) характерных значений. Ключ к пониманию природы масштабно-инвариантных явлений дает теория самоорганизованной критичности [1], демонстрирующая возникновение целостных свойств у открытых систем, состоящих из большого числа элементов, поведение которых подчиняется локальным нелинейным правилам. Базовым образом здесь служит куча песка, на которую по одной добавляют песчинки. Если песка в куче мало, то осыпание, вызванное добавленной песчинкой, скорее всего, не достигнет края кучи и песчинка останется в ней. Если песка много, возможно возникновение глобального осыпания, в результате которого большое количество песчинок свалится за край кучи. Равновесие между числом песчинок, добавляемых в кучу и покидающих ее, достигается при некотором критическом количестве песка, при котором возмущение может распространяться по куче сколь угодно далеко, в среднем не затухая и не разрастаясь. При этом описывающие его величины утрачиваются собственные характерные значения.

Исследователями предложен целый ряд самоорганизованно-критических моделей, из которых для данного обзора были взяты классические модели кучи пуска, представляющие собой клеточные автоматы на решетке размера $L \times L$. В ее ячейках находятся неотрицательные целые числа $z_{i, j}(0 \leqslant i, j<L)$, традиционно интерпретируемые как количество песчинок. Элементарное событие модели состоит из возмущения устойчивого состояния и процесса релаксащии. Устойчивым считается состояние, в котором для всех ячеек выполнено неравенство $z_{i, j}<z_{c}$, где $z_{c}-$ nорог устойчивости, одинаковый для всех ячеек. Возмущение производится путем увеличения на единицу значения в выбранной по определенным правилам ячейке $(i, j)$, что рассматривается как вброс в кучу песчинки: $z_{i, j}+=1$ (то есть к величине $z_{i, j}$ добавляется 1). Если в результате возмущения ячейка теряет устойчивость, то она опрокидывается, передавая некоторое количество песчинок своим соседкам согласно правилам модели. Это, в свою очередь, способно нарушить устойчивость соседних ячеек, приводя к развитию лавинь опрокидываний, продолжающейся до тех пор, пока все ячейки вновь не обретут устойчивость. После этого релаксационный процесс считается завершенным и дается старт следующему событию.

В настоящей работе рассматриваются 5 моделей типа кучи песка, правила опрокидывания которых, изображенные на рис. 1, демонстрируют различные комбинации особенностей:

- модель Дхара-Рамасвами (ДР) [2] - направленная, детерминированная, консервативная, абелева;

- модель Пастор-Саторраса-Веспиньяни (ПСВ) [3] - направленная, стохастическая, консервативная, квазиабелева;

- модель Федеров [4] - изотропная, детерминированная, диссипативная, неабелева;

- модель Maннь [5] - изотропная, детерминированная, диссипативная, квазиабелева; 

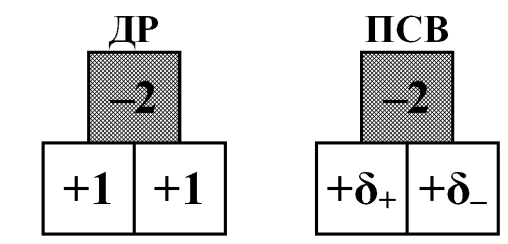

$$
z_{c}=2, z_{i, j}=\mathbf{2}
$$$$
z_{c}=2, z_{i, j}-=2
$$

$$
z_{i+1, j \pm 1 / 2}+=1
$$

$a$

$$
\begin{gathered}
\boldsymbol{z}_{i+1, j \pm 1 / 2}+=\boldsymbol{\delta}_{ \pm} \\
\boldsymbol{\delta}_{ \pm} \geq \mathbf{0}, \boldsymbol{\delta}_{+}+\boldsymbol{\delta}_{-}=\mathbf{2}
\end{gathered}
$$

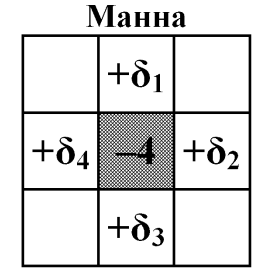

$z_{c}=4, z_{i, j}-=4$

$z_{i \pm 1, j \pm 1}+=\delta_{k}$

$\delta^{\delta_{k} \geq 0, \Sigma_{k} \delta_{k}=4}$

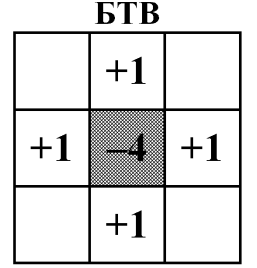

$z_{c}=4, z_{i, j}-=4$

$z_{i \pm 1, j \pm 1}+=1$

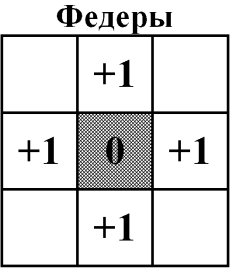

$z_{c}=4, z_{i, j}=0$

$z_{i \pm 1, j \pm 1}+=1$

Рис. 1. Опрокидывание неустойчивой ячейки (показана заливкой) в моделях кучи песка: $a$ - направленные модели Дхара-Рамасвами и Пастор-Саторраса-Веспиньяни; $\sigma$ - изотропные модели Манны, Бака-Танга-Визенфельда и Федеров

- модель Бака-Танга-Визенфельда (БТВ) [6,7] - изотропная, детерминированная, консервативная, абелева.

Для изотропных моделей (Федеры, Манна и БТВ) используется прямоугольная решетка, т.е. у каждой ячейки есть 4 соседки, имеющих с ней общую сторону, а для направленных (ДР и ПСВ) - треугольная, то есть у каждой ячейки есть 2 нижележащих соседки, имеющих с ней общие пол-стороны. Порог устойчивости ячеек равен числу соседок, т.е. $z_{c}=4$ для изотропных моделей и $z_{c}=2$ для направленных. Заметим, что перенесение направленных моделей на прямоугольную решетку, где у каждой ячейки будет 3 нижележащих соседки, имеющих с ней общие сторону или угол, не меняет существенно свойств моделей (для модели ДР это строго показано в работе [8], для модели ПСВ - продемонстрировано совпадением результатов в работах [3] и $[9,10]$, использующих разные типы соседства). Такие неканонические модификации далее не рассматриваются.

При опрокидывании неустойчивой ячейки она передает своим соседкам $z_{c}$; песчинок, причем в детерминированных моделях (ДР, Федеры и БТВ) песчинки раздаются поровну, то есть каждая соседка получает ровно по 1 штуке, тогда как в стохастических моделях (ПСВ и Манна) судьба каждой песчинки решается индивидуально и та попадает в случайно выбранную соседнюю ячейку.

В консервативных моделях (ДР, ПСВ, Манна, БТВ) при опрокидывании неустойчивой ячейки значение в ней уменьшается на величину $z_{c}$, вследствие чего число песчинок сохраняется, так что покидать систему они могут только через открытый край, а в диссипативной модели (Федеры) после опрокидывания число песчинок в неустойчивой ячейке обнуляется, так что в случае возникновения надкритического числа песчинок $z>z_{c}$ они способны покидать систему и в ее объеме.

Под абелевостью модели понимается независимость характеристик лавины от последовательности обработки неустойчивых ячеек. Этим свойством, очевидно, обладают детерминированные консервативные модели ДР и БТВ. Для абелевых моделей существует развитая теория, позволяющая аналитически определить ряд характеристик $[11,12]$. Если связать с каждой ячейкой стохастических консервативных моделей ПСВ и Манны свою последовательность случайных чисел, то формально эти модели тоже станут абелевыми. Однако их поведение существенно не изме- 
нится, если при опрокидывании неустойчивой ячейки изымать из нее и раздавать соседкам не пороговое количество песчинок, а все имеющиеся (для модели ПСВ работа [13], для модели Манны - результат автора). Однако после такой модификации правил модели уже не будут абелевыми, в силу чего они определены здесь как квазиабелевы. Инструментарий теории абелевых моделей к ним хотя и применим, но, скорее всего, ничего по-настоящему интересного узнать не позволит (иначе он бы работал и на неабелевых моделях). Диссипативная модель Федеров является неабелевой, так как для нее порядок опрокидывания ячеек существенно сказывается на развитии лавины.

Кроме правил опрокидывания неустойчивых ячеек необходимо описать правила вброса песчинок, инициирующих лавины, и правила выбытия песчинок, без которого невозможно достижение стационарного состояния системы.

Для направленных моделей (ДР и ПСВ) вброс песчинок осуществляется в случайно выбранную ячейку верхнего слоя. Нижний слой является открытым, то есть при опрокидывании его ячеек все изъятые из них песчинки покидают систему. Боковые края решетки отождествлены (периодические граничные условия).

Для диссипативной модели (Федеры) вброс осуществляется строго случайно по всей решетке. Краевые условия будут уточнены в ходе анализа этой модели.

Наконец, для консервативных изотропных моделей (Манна и БТВ) правила вброса песчинок непринципиальны (приводимые далее графики получены при вбросе, осуществляемом в одну из четырех центральных ячеек, выбираемую случайно). Краевые условия - открытые, то есть при опрокидывании прикраевой ячейки песчинки, выпавшие за край, покидают систему.

Целью изучения моделей является их решение, то есть определение показателей распределений различных характеристик лавин, из которых наиболее интересными являются:

- размер (количество произошедших опрокидываний) $N$;

- площяадь (число опрокинувшихся ячеек) $S$;

- периметр (число ячеек, получивших песчинку, но не опрокинувшихся, включая песчинки, выпавшие за край решетки) $C$;

- длительность (число шагов времени при одновременной обработке неустойчивых ячеек) $T$.

Кроме того, иногда полезным оказывается рассмотрение таких производных характеристик как средняя кратность опрокидывания $M=N / S$, активность $A=N / T$ и радиус (характерная линейная протяженность) области лавины $R \sim \sqrt{S}$.

Статья организована следующим образом. В разделе 1 описываются методы работы со степенными распределениями и облегчающий вычисление их показателей механизм несимметричного случайного блуждания. Раздел 2 посвящен моделям с анизотропным распространением активности, к числу которых относятся не только направленные модели ДР и ПСВ, но и изотропная модель Федеров. В разделе 3 на материале модели Манны демонстрируется инструментарий обращения с моделями, в которых распространение активности изотропно. В разделе 4 этот же инструментарий применен к модели БТВ. Завершается статья выводами, в которых суммируются и обобщаются ее результаты. 


\section{1. Степенные распределения вероятностей}

1.1. Скейлинговые свойства масштабно-инвариантных систем. Статистическим выражением масштабно-инвариантных свойств являются степенные распределения происходящих событий с плотностью вероятности вида

$$
u(x)=x^{-(1+\alpha)} \cdot g\left(x / x_{1}\right),
$$

где $\alpha$ - характеристический показатель; $g(\xi)$ - скейлинговая функиия, примерно постоянная при $\xi \ll 1$ и убывающая быстрее любой степени аргумента при $\xi \rightarrow \infty$. В силу масштабной инвариантности размер события $x_{1}$, крупного настолько, что оно уже не помещается в систему конечного размера $L$, растет как некоторая его степень

$$
x_{1} \sim L^{v}
$$

При этом доля событий, не помещающихся в систему, убывает с ростом ее размера как

$$
\operatorname{Prob}\left\{x>x_{1}\right\}=\int_{x_{1}}^{\infty} u(x) d x \cong \int_{L^{v}}^{\infty} x^{-(1+\alpha)} \cdot g\left(x L^{-v}\right) d x \sim L^{-\varepsilon},
$$

где $\varepsilon=\alpha v-$ универсальный показатель, величина которого не зависит от того, какая величина используется для его вычисления, поскольку если событие не помещается в систему, то оно не помещается сразу по всем своим характеристикам.

Сочетание формул (1) и (2) позволяет определить скейлинговое поведение и для среднего события

$$
\langle x\rangle=\int x \cdot u(x) d x \sim L^{\sigma}, \quad \sigma=v-\varepsilon .
$$

Первый и второй скейлинговые показатели $v$ и $\sigma$, связывающие характерные значения $x_{1}$ и $\langle x\rangle$ с обусловившим их появление конечным размером системы $L$, часто удается установить из общих соображений, что существенно облегчает теоретический анализ.

При одновременном рассмотрении различных характеристик $x$ и $y$ события зависимость между ними удобно описать условным средним некоторой степени $q>0$

$$
y(x)=\sqrt[q]{\left\langle y^{q}\right\rangle_{x}} \simeq c(q) \cdot x^{\gamma_{y x}} .
$$

Как правило, условное распределение $u(y \mid x)$ является узким, в результате чего показатель связи $\gamma_{y x}$ не зависит от выбора $q$, что позволяет при проведении расчетов с точностью до коэффициента считать связь таких величин функциональной. Далее используется степень $q=1$, то есть в качестве взаимозависимостей характеристик выступает условное среднее.

Сопоставление размеров крупных событий $y_{1} \sim x_{1}^{\gamma_{y x}}$ дает соотношение между показателем связи и скейлинговыми показателями

$$
\gamma_{y x}=v_{y} / v_{x}
$$

(C) А.В. Подлазов

Изв. вузов «ПНД», т. 24, № 4, 2016 
$\mathrm{C}$ другой стороны, формула преобразования вероятностей $u_{x}(x) d x=u_{y}(y) d y$ позволяет выразить показатель связи и через характеристические показатели

$$
\gamma_{y x}=\alpha_{x} / \alpha_{y},
$$

что в сочетании с формулой (5) приводит к тождеству

$$
\alpha_{x} v_{x}=\alpha_{y} v_{y}=\varepsilon
$$

подтверждающему независимость универсального показателя $\varepsilon$ от выбора рассматриваемой величины.

1.2. Случайное блуждание и показатели связи. На практике использование соотношения (5) предполагает условленность показателя связи для рассматриваемых величин. Определить его для систем, находящихся в самоорганизованнокритическом состоянии, позволяет учет природы последнего. Пусть имеется некоторая переменная $x$, характеризующая активность на шаге $t$. Ее изменение по шагам можно рассматривать как несмещенное случайное блуждание

$$
x_{t+1}=x_{t}+\delta_{t},
$$

где $\left\langle\delta_{t}\right\rangle=0$. В самом деле, при $\left\langle\delta_{t}\right\rangle<0$ лавина быстро затухнет, а при $\left\langle\delta_{t}\right\rangle>0$ может неограниченно развиваться, что приводит к появлению отрицательной обратной связи, удерживающей систему в состоянии с $\left\langle\delta_{t}\right\rangle=0$.

Пусть, кроме того, величина $x$ такова, что блуждание оказывается и некоррелированным, то есть $\left\langle x_{t} \cdot \delta_{t}\right\rangle=\left\langle x_{t}\right\rangle \cdot\left\langle\delta_{t}\right\rangle$. Тогда стандартная процедура возведения обеих сторон отображения (8) в квадрат с последующим усреднением приводит к формуле $\left\langle x_{t+1}^{2}\right\rangle-\left\langle x_{t}^{2}\right\rangle=\left\langle\delta_{t}^{2}\right\rangle$, которая при степенной зависимости среднеквадратичного приращения от номера шага и достигнутого значения

$$
\left\langle\delta_{t}^{2}\right\rangle \sim t^{\tau} x^{\xi},
$$

естественной для масштабно-инвариантных систем, позволяет вычислить показатель связи

$$
\gamma_{x t}=(\tau+1) /(2-\xi) .
$$

Как мы увидим далее, в ряде задач - при удачном выборе величины $x$ - показатели $\tau$ и $\xi$ находятся без труда.

Если $\tau=\xi=0$, то есть свойства блуждания не зависят ни от его результата, ни от прошедшего времени, то соотношение (10) дает показатель $\gamma_{x t}=1 / 2$, соответствующий обычной диффузии. В противном случае диффузия будет аномальной. Простейшим механизмом, лежащим в ее основе, является несимметричное блуждание с переменной длинной скачка. В частности, если $\delta_{t}= \pm \delta_{ \pm}$с вероятностями $p_{ \pm}$, то из нормировки $p_{+}+p_{-}=1$ и условия несмещенности $p_{+} \delta_{+}=p_{-} \delta_{-}$получается

$$
\left\langle\delta^{2}\right\rangle=\delta_{+} \delta_{-}
$$

Зависимость длин скачков $\delta_{ \pm}$от $t$ или $x$ может иметь сравнительно простое объяснение, что и позволяет перейти к формуле (9). 


\section{2. Модели с анизотропным распространением активности}

2.1. Направленные модели. Модель Дхара-Рамасвами была полностью решена сразу ее авторами в работе [2], а решение модели Пастор-Саторраса-Веспиньяни дано еще в работах $[9,10]$. Тем не менее, здесь развивается иной подход к этим моделям, следующий работе [14], отличительной чертой которого является возможность распространения и на более сложные модели.

В моделях ДР и ПСВ лавина распространяется строго сверху вниз, проходя каждый слой по одному разу. Поэтому длительность лавины $T$ совпадает с числом затронутых ею слоев. Различие между моделями состоит в том, что в модели ДР ни одна ячейка не опрокидывается более одного раза (то есть площадь лавины $S$ и ее размер $N$ совпадают), тогда как в модели ПСВ возможны многократные опрокидывания.

Длительность лавин, крупных настолько, что они уже не помещаются в систему, определяется, очевидно, числом имеющихся в решетке слоев

$$
T_{1}=L \quad \text { или } \quad v_{T}=1 .
$$

На каждом шаге в систему добавляется одна песчинка, поэтому в стационарном режиме в среднем одна песчинка будет ее и покидать. Единичное опрокидывание перемещает две песчинки на один слой, а для прохождения песчинки через всю систему необходимо перемещение на $L$ слоев. Таким образом, среднее количество опрокидываний во время лавины

$$
\langle N\rangle=L / 2 \quad \text { или } \quad \sigma_{N}=v_{N}-\varepsilon=1 .
$$

Будем характеризовать лавину на слое $t$ ее шириной (числом опрокинувшихся ячеек слоя) $w_{t}$ и активностью (числом произошедших на слое опрокидываний) $a_{t}$. Для модели ДР эти величины совпадают, тогда как в модели ПСВ они могут различаться.

При прохождении лавины по слоям ее ширина совершает случайные блуждания, смещение в которых никак не связано с номером слоя или текущей шириной, то есть в формуле (9) $\tau=\xi=0$ и соотношение (10) дает $\gamma_{w t}=1 / 2$. Типичная площадь лавины, достигшей слоя $T$, находится суммированием ширины по слоям $S(T)=\sum_{t=0}^{T} w_{t} \sim T^{\gamma_{w t}+1}$, или $\gamma_{S T}=3 / 2$, откуда, подставляя значение (12) в соотношение (5), получаем

$$
v_{S}=3 / 2 .
$$

Для модели ДР $v_{N}=v_{S}$ и $v_{C}=v_{T}$, то есть определены все скейлинговые показатели. Формула (13) дает значение универсального показателя $\varepsilon=1 / 2$, на основе чего из тождества (7) вычисляются и характеристические показатели распределений $\alpha_{c}=\alpha_{T}=1 / 2$ и $\alpha_{N}=\alpha_{S}=1 / 3$, завершает решение модели.

Для модели ПСВ необходимо сделать еще один шаг. Здесь при продвижении лавины по слоям случайные блуждания совершает и активность $a_{t}$, приращение которой является суммой случайных независимых случайных величин, взятой по ширине области лавины $w_{t}$. В самом деле, если с верхнего слоя в ячейку передается четное число частиц, то ее прохождение не меняет числа опрокидываний, а если нечетное, то при наличии в ней песчинки, число опрокидываний увеличивается на 
единицу, а при отсутствии - уменьшается [10]. Таким образом, $\left\langle\delta_{t}^{2}\right\rangle \sim w_{t} \sim t^{1 / 2}$, то есть в формуле (9) $\tau=1 / 2, \xi=0$ и соотношение (10) дает $\gamma_{a t}=3 / 4$. И по аналогии с формулой (14) получаем

$$
v_{N}=7 / 4 \text {. }
$$

Далее вновь с помощью значения (13) находится универсальный показатель $\varepsilon=3 / 4$, что почти завершает решение модели вычислением характеристических показателей $\alpha_{T}=3 / 4, \alpha_{N}=3 / 7$ и $\alpha_{S}=1 / 2$ из тождества (7).

Нерассмотренным остается только периметр лавины. Эта характеристика, которая далее сыграет ключевую роль при анализе моделей Манны и БТВ, а для моделей ДР и Федера является тривиальной, для модели ПСВ имеет не вполне четкий физический смысл, поскольку большая часть периметра оказывается внутри области лавины, а не на ее границе. Тем не менее, для полноты картины, следуя формальному определению периметра, посчитаем показатели и для него. Произвольная ячейка принадлежит периметру тогда и только тогда, когда она до лавины была пустой, вероятность чего пропорциональна ширине $w_{t}$, а в ее ходе получила ровно одну песчинку, вероятность чего обратно пропорциональна средней активности $a_{t} / w_{t}$. Таким образом, на слое оказывается порядка $w_{t}^{2} / a_{t} \sim t^{1 / 4}$ ячеек периметра. Соответственно, $v_{C}=5 / 4$ и $\alpha_{C}=3 / 5$.

2.2. Модель Федеров. Несмотря на строгую изотропию правил, модель Федеров демонстрирует направленную динамику, в силу чего может быть решена на основе того же подхода из работы [14], который был применен к моделям ДР и ПСВ. Однако сделать это, опираясь лишь на правила модели уже не удастся - необходимо предварительное изучение ее свойств и построение модели промежуточного уровня.

Может показаться, что в силу наличия в системе диссипации для достижения стационарного состояния открытый край не нужен. Однако, как показывает моделирование, если отождествить противоположные края решетки, свернув ее в тор, то система в какой-то момент впадает в бесконечный цикл. То есть, то количество песчинок в системе, которого достаточно для поддержки сколь угодно длительной лавины, оказывается уже недостаточным для возникновения диссипации.

Примечательно, что во время бесконечной лавины среднее заполнение ячеек $\langle z\rangle=z_{\infty}=2$ точно и без дисперсии. Объясняется данное значение устройством бесконечной лавины, которая представляет собой один или несколько фронтов опрокидывания, циклически обходящих решетку. При этом каждая ячейка, потерявшая устойчивость, при опрокидывании должна в среднем вернуть назад (туда, откуда фронт уже ушел) столько же песчинок, сколько она передает вперед (туда, куда идет фронт). А вперед передается столько песчинок, сколько в среднем не хватает ячейке до потери устойчивости. В сочетании с отсутствием диссипации два указанных обстоятельства и приводят к значению $z_{\infty}=z_{c} / 2=2$.

Далее мы будем полагать верхний и нижний края решетки открытыми, а левый и правый отожествим, свернув ее в вертикальный цилиндр, как и для моделей ДР и ПСВ. Такая топология наиболее удобна для анализа, поскольку система становится квазиодномерной, распадаясь на слои ячеек, находящихся на одинаковом расстоянии от края.

У каждой песчинки, попавшей в систему, есть два пути ее покинуть: диссипация при опрокидывании ячейки с надкритическим числом песчинок или падение за 
край при опрокидывании краевой ячейки. Как показывает компьютерное моделирование, второй путь значим только для событий, начинающихся в непосредственной близости от края. По мере удалении точки вброса от края число песчинок, выпавших за него, стремительно уменьшается (примерно в 10 раз на 10 слоях). Это обусловлено неконсервативностью правил, ограничивающей длину свободного пробега песчинок по решетке, в результате чего они покидают ее на сравнительно небольшом расстоянии от места вброса (в силу чего в правила и было введено требование его пространственной равномерности). Поэтому почти все песчинки выбывают в результате диссипации, а за край падают только те немногие, которым удается его достичь.

В результате возникает сложная пространственная структура заполнения, изображенная на рис. 2. За исключением прикраевого слоя и области в середине решетки всюду $\langle z\rangle>z_{\infty}$. Нарушение этого неравенства ведет к прекращению активности, а значит, и диссипации, в результате чего приток песчинок начинает преобладать над их оттоком, увеличивая заполнение. Общий вид его зависимости от номера слоя тоже вполне понятен. С одной стороны, вдалеке от края величина $\langle z\rangle$ должна убывать, приближаясь к значению $z_{\infty}$, реализующемуся в системе без краев. А с другой - на самом краю из-за падения песчинок за него $\langle z\rangle$ тоже мало. Где-то между этими двумя минимумами должен быть и максимум. Причем он располагается очень близко к краю (всего лишь на 4-м от него слое - см. левую врезку на рис. 2) в силу отмеченного выше экспоненциального сокращения шансов песчинки выпасть за край при удалении от него.

В таких условиях открытый край становится источником активности. Чем ближе к нему вброшена инициирующая песчинка, тем выше заполнение и лучше усло-

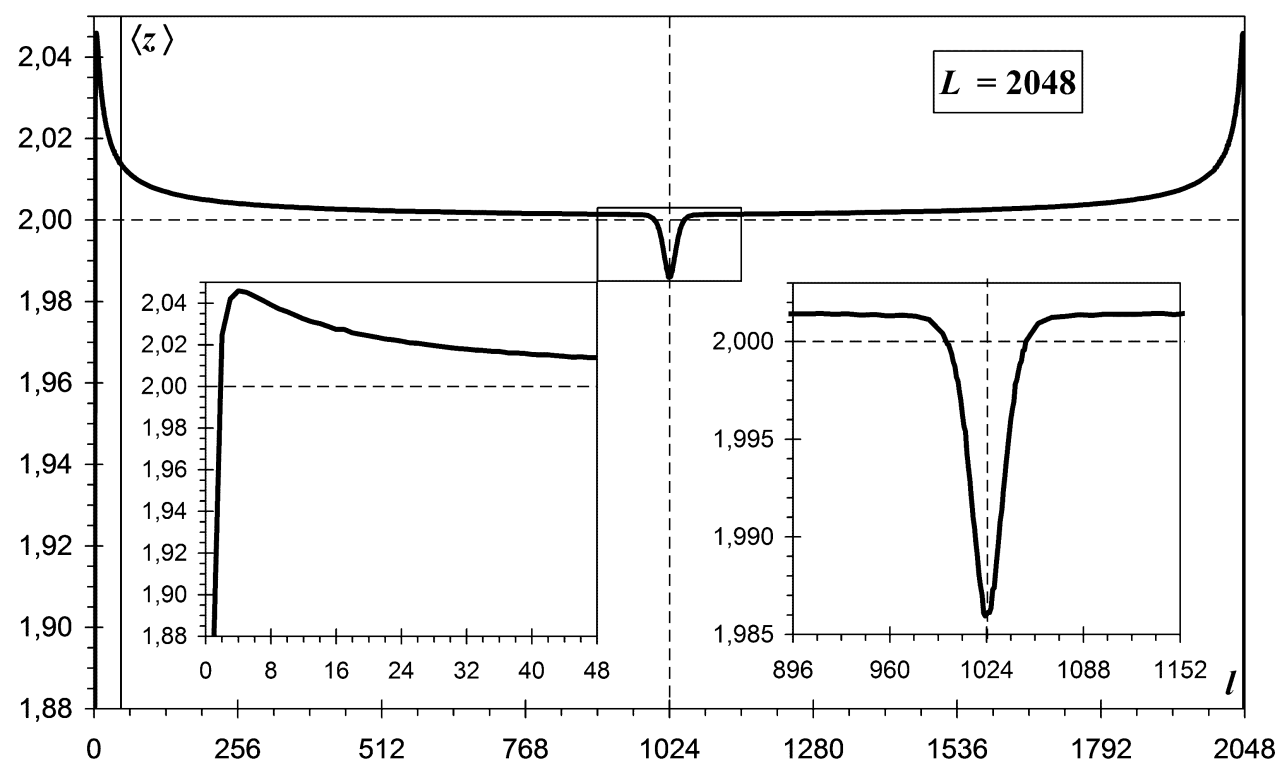

Рис. 2. Среднее заполнение ячеек по слоям решетки в модели Федеров. Заполнение максимально вблизи края, а по мере по мере удаления от него уменьшается, стремясь по гиперболе к предельному значению $z_{\infty}$, но в середине решетки проседает до значений, меньших $z_{\infty}$. На врезках увеличена области, обведенные рамочками: на левой - прикраевая область с максимумом заполнения, на правой центральная с его минимумом. Слои нумеруются от одного края до другого (а не до середины, как в тексте)

(C) А.В. Подлазов

Изв. вузов «ПНД», т. 24, № 4, 2016 
вия для развития лавины. Поэтому самые крупные лавины, происходящие в системе, начинаются около ее края и завершаются около ее середины. Возникновение крупной лавины, идущей в обратную сторону, маловероятно. Такое поведение модели в самоорганизованно критическом состоянии уместно характеризовать как спонтанную анизотропию. Она связанна с распространением краевых эффектов на всю систему (точнее говоря, на ближайшую к данному краю ее половину). Приближенно можно считать, что лавины идут только вглубь решетки, принимая вид одного или изредка нескольких последовательных фронтов опрокидывания. При этом длительность лавины оказывается соизмерима с числом пройденных ею слоев, а размер с площадью.

Для модели Федеров показатели (12) и (13) сохраняются, хотя если величина первого очевидна в силу анизотропии распространения активности, то природа второго существенно меняется. Можно показать, что среднее число опрокидываний в слое $l$, вызванных вбросом инициирующей песчинки на слой $l_{0}$, для системы достаточно большого размера $L$ удовлетворительно приближается формулой

$$
\left\langle N\left(l, l_{0}\right)\right\rangle \sim \frac{\theta\left(l-l_{0}\right)}{L}
$$

где $\theta$ - функция Хевисайда, а слои нумеруются от ближайшего края к середине. Вид числителя этой формулы означает, что количество опрокидываний на слое зависит только от его расположения относительно слоя вброса, но не зависит напрямую ни от номеров слоев, ни от расстояния между ними. Иными словами, лавины в своем развитии не испытывают систематической тенденции ни к разрастанию, ни к угасанию, как и должно быть в самоорганизованно-критическом состоянии. Знаменатель формулы (15) определяется из того соображения, что среднее число опрокидываний, происходящих на слое за время, соответствующее одному вбросу на него инициирующей песчинки (то есть за $L$ шагов), не зависит от размера системы. Соответственно, за один шаг должна получаться величина, обратно пропорциональная $L$, в чем можно убедиться непосредственно, а именно:

$$
\langle N(l)\rangle=2 \int_{0}^{L / 2}\left\langle N\left(l, l_{0}\right)\right\rangle d l_{0} \sim l / L
$$

А повторное интегрирование и дает зависимость $\langle N(l)\rangle=2 \int_{0}^{L / 2}\langle N(l)\rangle d l \sim L$, воспроизводящую значение (13).

Следует отметить, что спонтанная анизотропия модели Федеров, хоть и является очень похожей на анизотропию, изначально заложенную в правила моделей ДР и ПСВ, все-таки отличается тем, что имеет место лишь в глобальном масштабе. Локальная динамика остается изотропной, что приводит к такому важному явлению, отсутствующему в направленных моделях, как возвращение активности на уже пройденные лавиной слои, которое и обуславливает различия показателей распределений для моделей ДР и Федеров.

Если в случае модели ДР можно строго показать, что область лавины является односвязной и не имеет дырок, то в случае модели Федеров односвязность, вообще говоря, ниоткуда не следует. Однако возникновение дырок в этой модели является, по-видимому, событием весьма маловероятным. В ходе компьютерного исследования 
модели автору ни разу не встретились дырявые лавины, зато неоднократно наблюдалось зарастание уже, казалось бы, вполне оформившейся дырки. Поэтому можно считать, что дырок нет, а изменение ширины области лавины $w$ обусловлено исключительно блужданием, совершаемым ее границей при изменении номера слоя $l$.

Данное блуждание оказывается несимметричным. Так, если происходит уменьшение ширины области лавины, ее граница сдвигается внутрь один раз. А если происходит увеличение ширины, то граница может сдвигаться вовне несколько раз из-за возвращения активности на слой. Успешному, то есть приводящему к новым опрокидываниям, возвращению активности способствует медленный рост ширины области лавины по слоям, поскольку вдоль менее крутой границы активность может возвратиться на большее число слоев. Схематично эта ситуация представлена на рис. 3.

Можно считать, что длина скачка, совершаемого границей вовне, пропорциональна числу слоев, на которых активность может за один раз вернуться, сдвигая границу, то есть $\delta_{+} \sim \delta l / \delta s$, тогда как длина скачка вовнутрь $\delta_{-}=$const. Отсюда с помощью формулы (11) находятся показатели в формуле (9) $\tau=1, \xi=-1$ и соотношение (10) дает $\gamma_{w t}=2 / 3$, в связи с чем на смену значению (14) приходит

$$
v_{S}=5 / 3 .
$$

Как и для модели ДР здесь $v_{N}=v_{S}$ и $v_{C}=v_{T}$, поэтому все последующие шаги аналогичны. Универсальный показатель в модели Федеров $\varepsilon=2 / 3$, а характери-
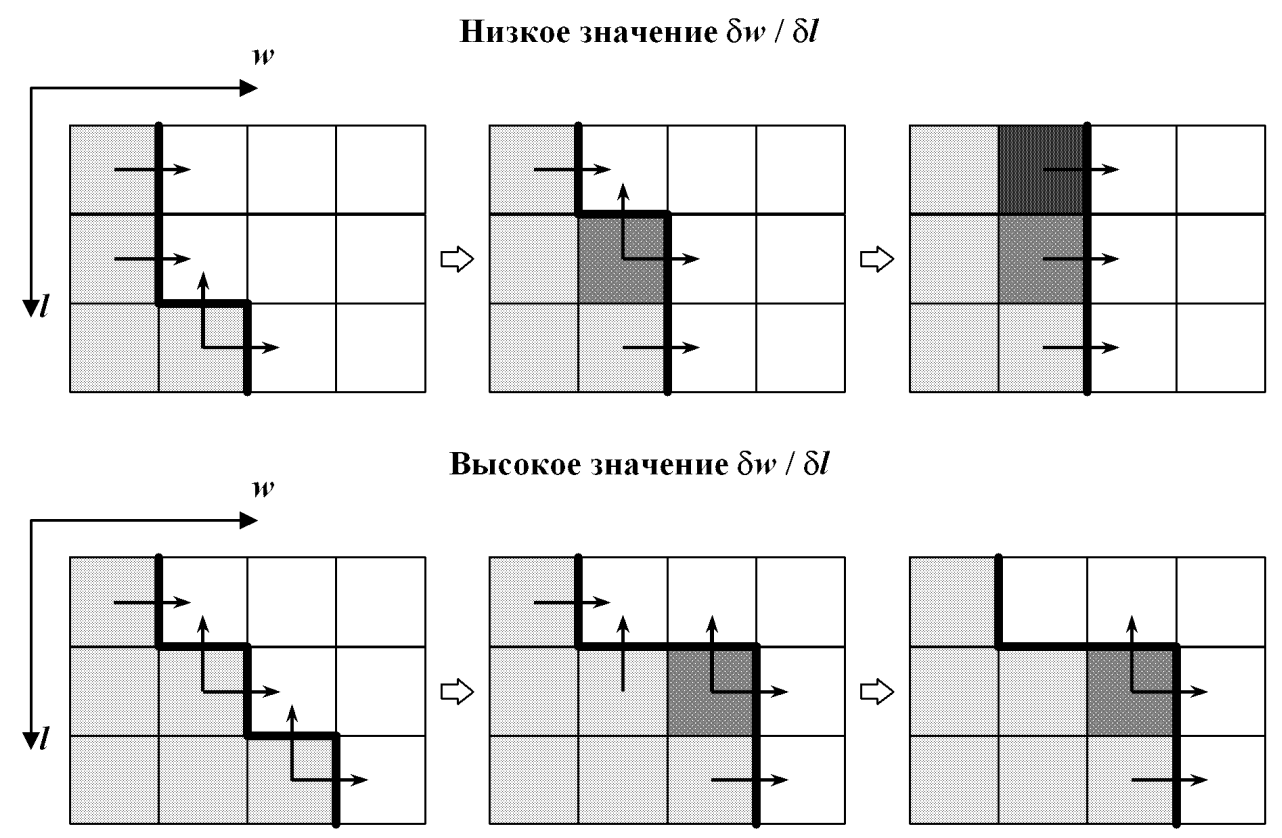

Рис. 3. Пример возвращения активности. Стрелками показана передача песчинок при опрокидывании ячеек. Светлой заливкой отмечены ячейки, опрокинувшиеся при первом же прохождении лавины по слоям, промежуточной - ячейка, опрокидывание которой происходит при возвращении активности на один слой, а темной - ячейка, опрокидывание которой происходит (вверху) или не происходит (внизу) при возвращении активности на два слоя. Жирной линией показана граница области лавины. Чем быстрее она смещается вовне при движении по слоям, тем ниже шансы на успешное возвращение активности 


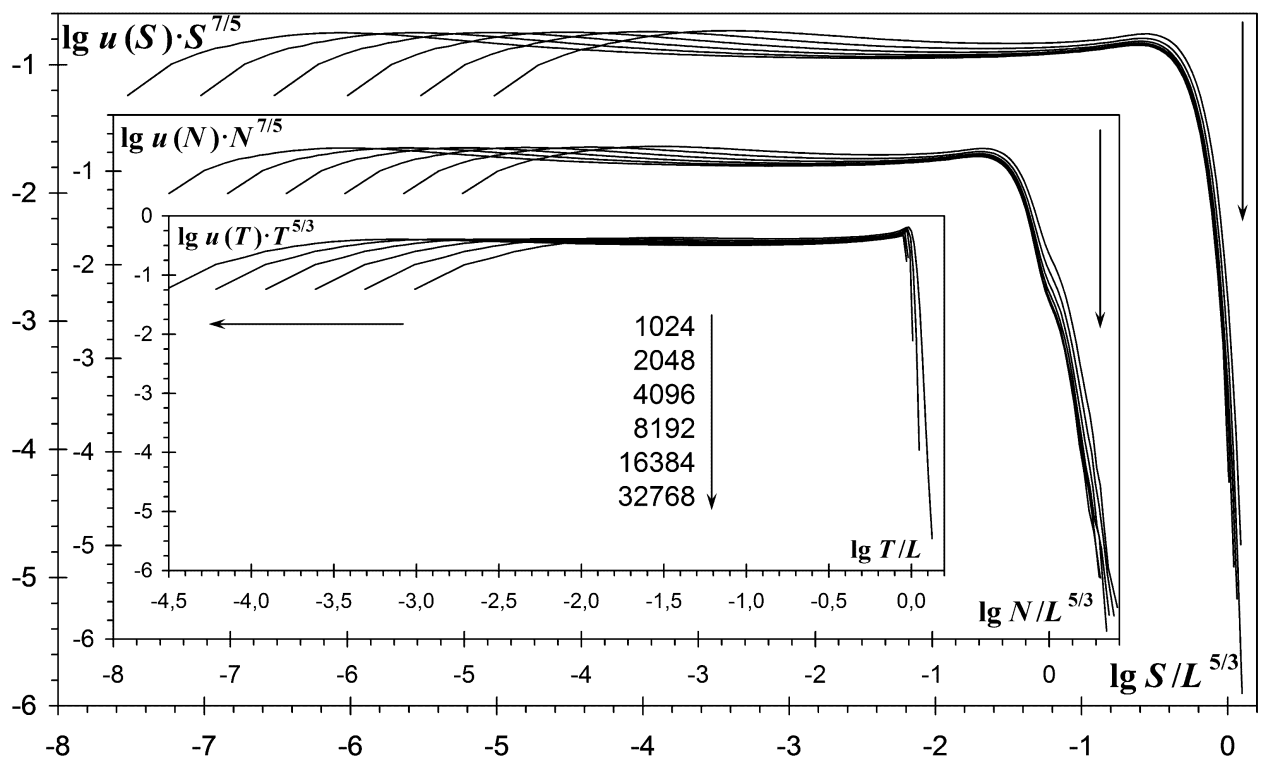

Рис. 4. Распределения лавин в модели Федеров. За счет домножения плотности распределения на $x^{1+\alpha}$ графики приобретают горизонтальный участок, который соответствует области промежуточной асимптотики, где распределение имеет степенной вид. При этом за счет деления аргумента на $L^{v}$ получается скейлинговая функция распределения из формулы (1), к которой графики приближаются по мере увеличения $L$ (здесь и далее указано стрелками). Даже для решеток очень большого размера наложение графиков оказывается неполным, что говорит о существенной роли краевых эффектов

стические $\alpha_{C}=\alpha_{T}=2 / 3$ и $\alpha_{N}=\alpha_{S}=2 / 5$. Рис. 4 подтверждает правильность найденных значений.

Завершим обсуждение модели Федеров, пояснением природы рва заполнения (см. правую врезку на рис. 2), возникающего посередине решетки. Ров заполнения служит яркой и наглядной иллюстрацией самоорганизационного возникновения целостных свойств, то есть свойств, имеющихся у системы, но в принципе отсутствовавших у ее составных частей. Правила модели вполне локальны и оперируют лишь соседними ячейками. Более того, дистанция, проходимая каждой отдельной песчинкой за время ее пребывания в системе, ограничена несколькими десятками ячеек. Тем не менее, система произвольного размера находит свою середину. Ров отрывается постоянным потоком песчинок, направленным к ближайшему краю решетки. Величина потока находится дифференцирование формулы (16):

$$
\frac{d}{d l}\langle N(l)\rangle \sim \frac{1}{L}
$$

Структура заполнения решетки вполне определяется особенностью распределения активности по слоям. Так, ров отрывается потоками песка, направленными от середины решетки к ее краям. При этом следует заметить, что за счет флуктуаций заполнения лавины могут немного перехлестывать за середину решетки, вынося там песчинки на другую сторону рва, то есть перемещая их сразу по направлению к обоим краям. В противном случае, рва бы не образовывалось, поскольку правила модели изотропны и суммарный перенос песка любой лавиной равен нулю. 
Примечательным отличием модели Федеров от рассматриваемых консервативных моделей является противонаправленность распространения активности и переноса песчинок. Активность идет от края в глубину, а песчинки перемещаются из глубины к краю. В консервативных моделях эти процессы сонаправлены.

\section{3. Модель Манны}

Исследование модели Манны дается на основе работ [15-17]. Эта модель существенно сложнее моделей с анизотропным распространением активности. Однако, как и в их случае, из правил модели Манны легко можно определить два скейлинговых показателя.

Во-первых, площадь области лавины, очевидно, ограничена площадью решетки:

$$
S_{1} \sim L^{2} \text { или } v_{S}=2 .
$$

Во-вторых, движение каждой отдельно взятой песчинки по решетке представляет собой случайное блуждание. Чтобы покинуть решетку, добравшись от ее середины до открытого края, песчинке надо пройти дистанцию порядка $L$ ячеек, что требует порядка $L^{2}$ шагов. Каждая лавина начинается добавлением одной песчинки в систему, поэтому и покидать ее в стационарном состоянии будет в среднем одна песчинка. Таким образом,

$$
\langle N\rangle \sim L^{2} \text { или } \sigma_{N}=v_{N}-\varepsilon=2 .
$$

Отсюда немедленно находится характеристический показатель для средней кратности опрокидывания $M=N / S$. Из равенства показателей (17) и (18) получаем $v_{M}=v_{N}-v_{S}=\varepsilon$, что в сочетании с тождеством (7) дает

$$
\alpha_{M}=1 .
$$

По аналогии с формулой (3) при любом значении $v_{M}$ можно записать

$$
\langle M\rangle=\int M^{-1} \cdot g\left(M / L^{v_{M}}\right) d M \sim \int^{L^{\nu_{M}}} d M / M \sim \log L,
$$

что легко проверяется по результатам моделирования (см. рис. 12 далее).

Единичный характеристический показатель очень удобен и при проведении выкладок, так как соотношение (6) упрощается до тождества

$$
\alpha_{x}=\gamma_{M x} .
$$

Заметим, что в случае моделей с анизотропным распространением активности такая же ситуация имела место для средней активности $A=N / T$, для которой в силу равенства показателей (12) и (13) получалось $\alpha_{A}=1$. Но если для решения этих моделей такой результат был излишним, то модель Манны далее будет решена с помощью значения (19) тремя разными способами. Подобная избыточность позволяет не только продемонстрировать общую технику исследования самоорганизованнокритических систем и убедиться в адекватности построенной модели промежуточного уровня, но и подготовить почву для изучения модели БТВ, которая еще сложнее, чем модель Манны. 
3.1. Пространственная декомпозиция области лавины. Область лавины представляется как последовательность слоев опрокидывания, внутри которых его кратность примерно одинакова, как показано на рис. 5. Для модели БТВ, правила которой строго детерминированы, такое описание было бы строгим (см. рис. 10 далее). Однако для модели Манны в силу стохастичности ее правил оно является лишь приближением. Тем не менее, поскольку основанные на нем расчеты находятся в прекрасном согласии с результатами моделирования, точность такого приближения следует считать удовлетворительной.

Слои области лавины нумеруются из ее глубины наружу, начиная с единицы. То есть, если максимальная кратность опрокидывания для рассматриваемой лавины равна $m$, то она состоит из слоев с номерами $0 \leq k<m$, на которых произошло $(m-k)$ опрокидываний.

В силу масштабной инвариантности критического состояния характерная величина внешнего радиуса $k$-го слоя дается степенной зависимостью

$$
r_{k} \sim k^{\gamma}
$$

в которой коэффициент пропорциональности полагается не зависящим от $m$. Иначе говоря, по мере развития лавины при появлении новых слоев границы уже имеющихся не испытывают систематического смещения в какую-либо сторону, подвергаясь лишь флуктуациям. Это предположение является очень сильным и неочевидным. Как будет показано далее, будучи справедливым для модели Манны, оно не выполняется для модели БТВ.

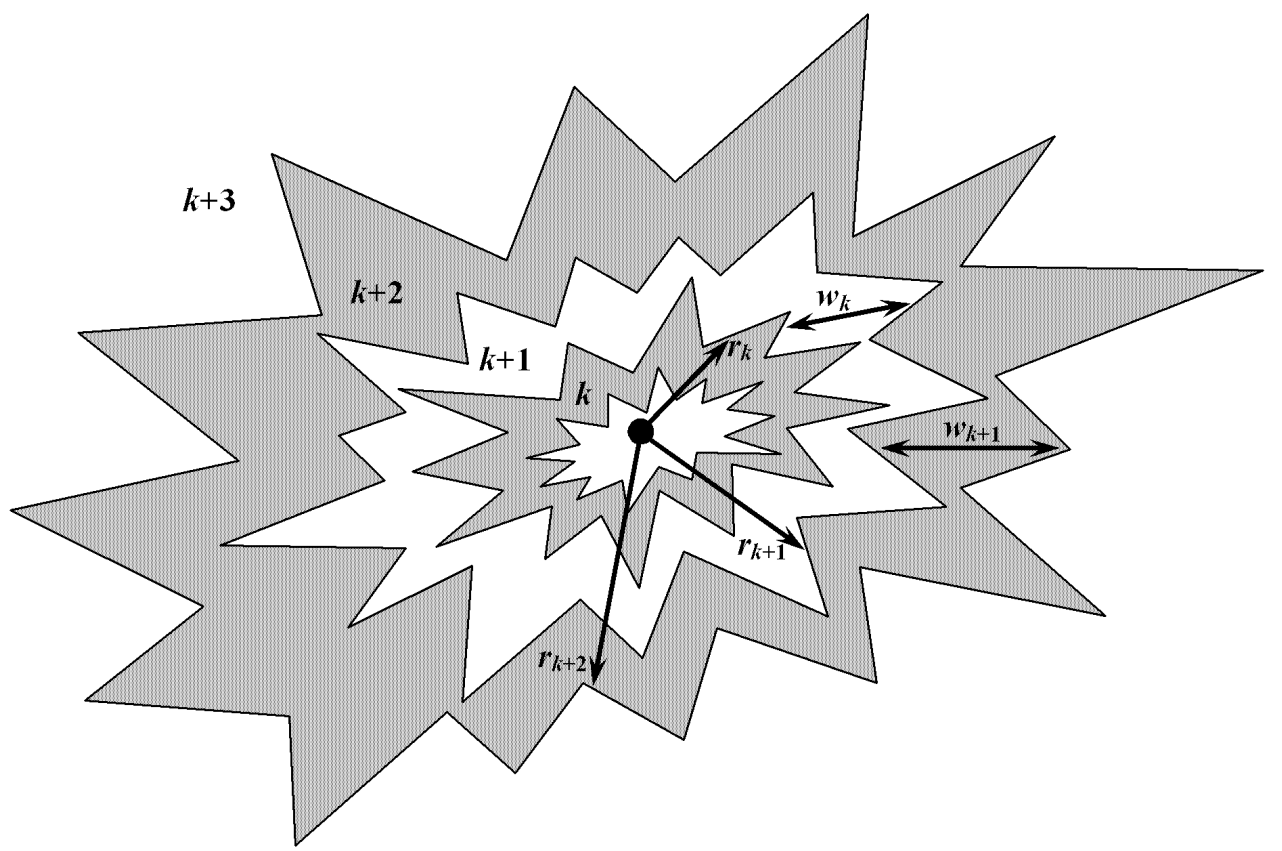

Рис. 5. Схематичное представление слоев опрокидывания. Увеличение номера слоя соответствует уменьшению кратности опрокидывания 
Характерная ширина $k$-го слоя

$$
w_{k} \cong \frac{d r_{k}}{d k} \sim k^{\gamma-1} \sim r_{k}^{1-1 / \gamma},
$$

а его площадь

$$
s_{k} \sim r_{k} w_{k} \sim k^{2 \gamma-1} \sim r_{k}^{2-1 / \gamma}
$$

Определим периметр слоя как длину его внешней границы. Она не является гладкой линией, образуя складки, соизмеримые по размеру с шириной слоя (23) (см. рис. 5). Поэтому его периметр не пропорционален радиусу (22). Назовем фронтом слоя усредненное положение его внешней границы. Поскольку усреднение сглаживает ее складки, длину фронта уже можно считать пропорциональной радиусу слоя. Количество складок границы определяется длиной фронта, что позволяет записать для периметра выражение

$$
c_{k} \sim r_{k} w_{k} \sim s_{k} \sim k^{2 \gamma-1} \sim r_{k}^{2-1 / \gamma} .
$$

Внутри слоя ячейки (в среднем) отдают соседям и получают от них одно и то же число песчинок. Однако на границе слоев этот баланс нарушается, так как ячейки внутреннего слоя отдают больше, чем получают от ячеек внешнего. То есть, количество песчинок, передаваемых слоем вовне, определяется его периметром (25), в каждой точке которого происходит передача песчинки.

Введем понятие активных частиц, под которыми будем понимать песчинки, которые в ходе лавины покидают слой без компенсации. Количество активных частиц на $k$-ом слое равно разнице числа отданных наружу и полученных изнутри песчинок:

$$
a_{k} \cong \frac{d c_{k}}{d k} \sim k^{2 \gamma-2} \sim r_{k}^{2-2 / \gamma} .
$$

Активные частицы перемещаются из глубины области лавины к ее периферии, тогда как остальные песчинки - в случайном направлении. Таким образом, развитие лавины представляет собой сочетание направленного и ненаправленного движений. Подобное разделение имеет смысл только в критическом состоянии. В докритическом состоянии активные частицы будут утрачивать этот статус, не достигая границ области лавины, а в сверхкритическом даже вынос вовне всех активных частиц не будет приводить к завершению лавины из-за продолжающегося появления новых активных частиц.

Переносом активных частиц определяется продвижение фронтов вовне, скорость которого пропорциональна числу активных частиц, приходящихся на единицу длины фронта

$$
v_{k} \sim \frac{a_{k}}{r_{k}} \sim k^{\gamma-2} \sim r_{k}^{1-2 / \gamma} .
$$

Следовательно, время, которое фронт пребывает в пути с момента своего появления, составляет

$$
t_{k} \sim r_{k}^{2 / \gamma} \sim k^{2} .
$$

С помощью формул (26) и (27) запишем поток частиц через $k$-й слой $j_{k}=v_{k} a_{k} \sim r_{k}^{3-4 \gamma}$. Он не должен зависеть ни от номера слоя, ни от числа слоев, ибо он выносит ту самую песчинку, добавлением которой инициирована лавина. Таким образом,

$$
\gamma=4 / 3
$$

(C) А.В. Подлазов

Изв. вузов «ПНД», т. 24, № 4, 2016 
Формула (24) позволяет выразить размер $N=\sum_{k=0}^{m}(m-k) s_{k} \sim m^{2 \gamma+1}$ и площадь $S=\sum_{k=0}^{m} s_{k} \sim m^{2 \gamma}$ лавины через максимальную кратность опрокидывания. Таким образом, средняя кратность опрокидывания $M$ отличается от максимальной $m$ лишь коэффициентом, откуда на основе значения (29) и тождества (21) находим $\alpha_{N}=\gamma_{M N}=3 / 11$ и $\alpha_{S}=\gamma_{M S}=3 / 8$. Подставляя в формулы (25) и (28) в качестве номера слоя $m$, аналогичным образом получаем $\alpha_{C}=\gamma_{M C}=3 / 5$ и $\alpha_{T}=\gamma_{M T}=1 / 2$.

Наконец, из соотношений (7) и (18) определяем сначала значения показателей $v_{N}=11 / 4$ и $\varepsilon=3 / 4$, а затем и $v_{M}=3 / 4, v_{C}=5 / 4$ и $v_{T}=3 / 2$, завершая тем самым решение модели.

Сопоставление полученных результатов с компьютерным экспериментом представлено на рис. 6. Совпадение является очень хорошим для всех показателей, кроме скейлингового показателя для длительности. Представляется весьма вероятным, что зависимости между различными характеристиками событий, иногда могут иметь не чисто степенной вид (4), а приобретать к нему логарифмические поправки, связанные с недостаточной узостью условных распределений. При размерах систем, доступных для моделирования, логарифмическая функция неотличима от степенной, имеющей показатель в пределах нескольких сотых долей. Это может приводить к возникновению систематических погрешностей при экспериментальном определении показателей, в результате отклоняющихся от строгого соответствия формулам (5) и (6). Однако практика показывает, что подобная неприятная ситуация никогда не возникает для величин, имеющих зафиксированный правилами скейлинговый показатель $v$ или $\sigma$. Именно на них следует ориентироваться, проверяя решение нало-

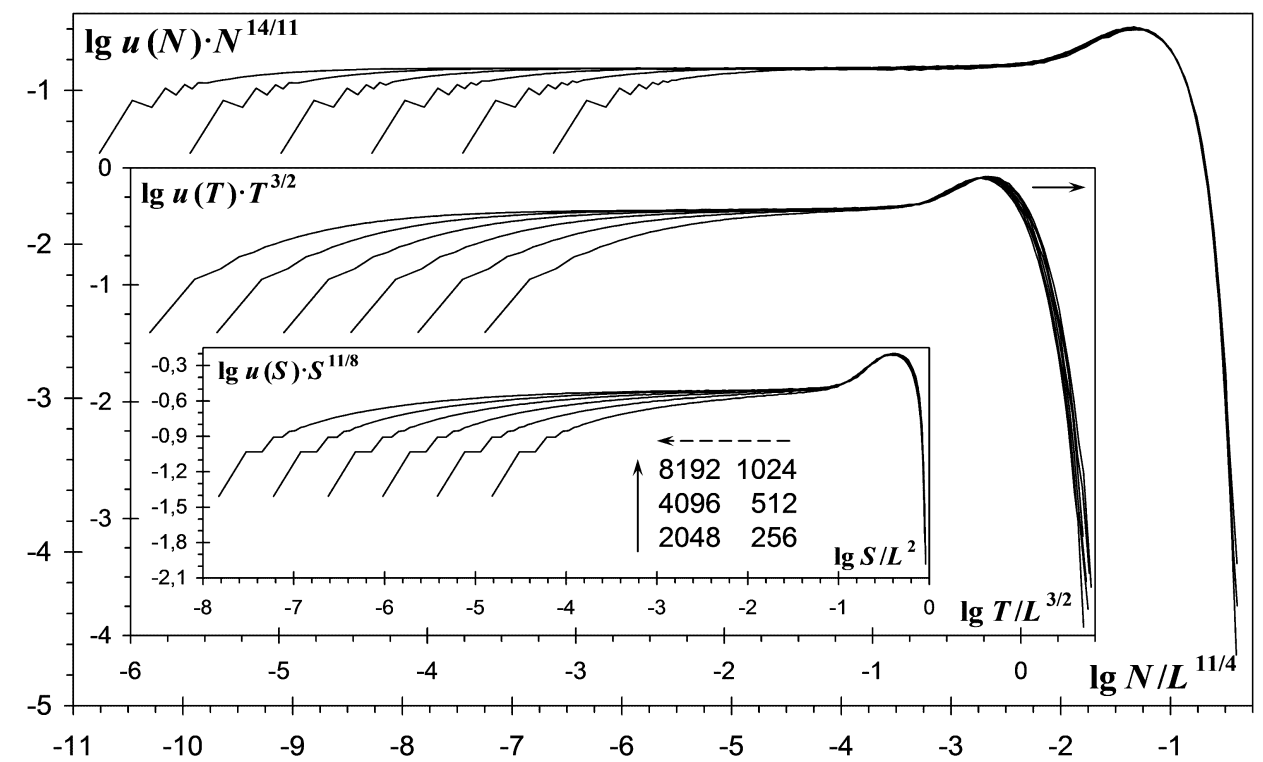

Рис. 6. Распределения лавин в модели Манны. Рисунок аналогичен рис. 4. Здесь наложение графиков даже для решеток не столько большого размера, как в случае модели Федеров, заметно лучше, что свидетельствует о меньшей роли краевых эффектов. Исключением является лишь распределение лавин по длительности $T$, для которого картина существенно хуже, чем для распределений по размеру $N$ и площади $S$. Чтобы исправить ситуацию, пришлось бы увеличить теоретическое значение $v_{T}$ на несколько сотых 
жением отмасштабированных графиков плотности. В силу формул (17) и (18) такими характеристиками для модели Манны являются площадь лавины и ее размер, для которых теоретические показатели идеально проходят экспериментальную проверку.

Аналогичная ситуация имеет место и для модели ПСВ с показателями, описывающими площадь и периметр. При их экспериментальном определении тоже возникают систематические погрешности до нескольких сотых, хотя показатели, описывающие размер и длительность, находятся с высокой точностью, обеспеченной привязкой $v_{T}$ и $\sigma_{S}$ к правилам модели.

3.2. Сведение к случайному блужданию. Как и в случае модели ПСВ, тоже имеющей стохастические правила, здесь потребуется рассмотрение двух процессов случайного блуждания. С первым из них связано изменение кратности опрокидывания $M$ с течением времени $T$. Для этого процесса в формуле (9) $\tau=\xi=0$ и соотношение (10) в сочетании с тождеством (21) дают значение $\alpha_{T}=\gamma_{M T}=1 / 2$, через который далее будут выражены все остальные показатели модели.

Второе блуждание является более сложным процессом и отвечает за изменение активности $A$, которое происходит при продвижении фронта лавины вовне. При этом приращение активности возникает как сумма независимых случайных величин, взятая по всему фронту лавины, длина которого пропорциональна радиусу ее области $R$. Таким образом, чтобы выразить активность через время, надо сначала выразить радиус через время.

Не вводя новых обозначений, запишем $\gamma_{r t}=\tau$, имея в виду пока неизвестный показатель из формулы (9). В ней $\xi=0$ (количество ненулевых слагаемых в рассматриваемой сумме пропорционально, а приращение радиуса за один шаг обратно пропорционально текущей активности, так что ее вклады в величину приращения взаимокомпенсируются), так что соотношение (10) дает $\gamma_{a t}=(\tau+1) / 2$. Отсюда получаем $\gamma_{N T}=\gamma_{a t}+1=(\tau+3) / 2$ и (с учетом соотношения (6)) $1 / \alpha_{N}=\tau+3$. С другой стороны, в силу компактности области лавины $\gamma_{S R}=2$, что приводит к значению $\gamma_{S T}=\gamma_{S R} \cdot \gamma_{R T}=2 \tau$, позволяющему с помощью формул (6) и (7) определить универсальный показатель $\varepsilon=1 / 2 \tau$. Наконец, подстановка найденных значений в выражение (18), переписанное посредством тождества (7) в виде $1 / \alpha_{N}-1=2 / \varepsilon$, образует уравнение, из которого вычисляется значение $\tau=2 / 3$, а через него легко рассчитываются все остальные показатели модели.

Единственным отличием в результатах между этим разделом и предыдущим является то, что там фигурировал периметр области лавины, а здесь - ее активность. Однако эти две величины характеризуются одними и теми же показателями. И это неслучайно. Если просуммировать определение (26) по слоям лавины, то общее количество активных частиц совпадет с ее периметром. Иначе говоря, активные частицы, совершающие направленное движение, по завершении лавины и образуют ее периметр, или, что то же самое, вынос всех активных частиц на периферию области лавины и ведет к ее завершению.

3.3. Ланжевеновское уравнение. Рассмотрим изменение продвижения границы области лавины как динамический процесс. В момент времени $t$ (заметим, что это не обязательно модельное время) положение границы в полярных координатах определяется направлением $\varphi$ и радиусом $r(\varphi, t)$, отсчитываемыми от точки вброса песчинки, инициировавшей лавину. Такое описание является приближенным, так как 
не позволяет учитывать загибы границы, однако дальнейшее совпадение результатов выкладок и эксперимента позволяет считать его удовлетворительным.

В силу масштабной инвариантности критического состояния средний радиус области лавины зависит от времени степенным образом:

$$
r(t)=\langle r(\varphi, t)\rangle \sim t^{1 / z},
$$

где $z$ - динамический показатель.

Длина границы возрастает быстрее ее радиуса, набираясь преимущественно на ее выступах. Поэтому интерес представляет отклонение радиуса по данному направлению от среднего значения

$$
h(\varphi, t)=r(\varphi, t)-r(t) .
$$

Мерой неровности границы служит ее ширина

$$
w(t)=\sqrt{\left\langle h^{2}(\varphi, t)\right\rangle} \sim r^{\chi}(t),
$$

где $\chi$ - показатель шероховатости.

Изменение положения границы обусловлено двумя основными процессами. Первый - боковое распространение ее выступов, второй - их возникновение за счет вовлечения в лавину новых ячеек с высоким заполнением. Таким образом, динамику границы можно описать стохастическим уравнением переноса следующего вида:

$$
\frac{\partial h(\varphi, t)}{\partial t}=V \cdot\left|\frac{\partial h(\varphi, t)}{r(t) \partial \varphi}\right|+\eta,
$$

где $V$ - скорость продвижения склонов выступов, происходящего в обоих (отсюда модуль) тангенциальных направлениях, а шумовой член $\eta$ описывает рост выступов в радиальном направлении, обусловленный действием случайных факторов.

Прежде чем конкретизировать вид этих величин, заметим, что масштабноинвариантная запись вида (30) предполагает, что все члены уравнения (31) при пространственном огрублении должны оставаться одного и того же порядка величины или становиться пренебрежимо малы. Иными словами, при одновременной перенормировке переменных

$$
r \rightarrow b \cdot r, \quad t \rightarrow b^{z} \cdot t, \quad h \rightarrow b^{\chi} \cdot h
$$

вид уравнения (31) должен сохраняться неизменным за исключением, возможно, выпадения несущественных членов (таковы, например, все члены со старшими производными, учет которых в правой части уравнения (31) должен обеспечить его корректность). Поэтому при определении поведения $V$ и $\eta$ мы будем ориентироваться лишь на те факторы, которые остаются существенными при пространственном огрублении.

Тангенциальное продвижение склонов выступов является сильно коррелированным процессом, поскольку на склоне находятся ячейки, получившие дополнительные песчинки. Опрокидывание любой из них с высокой вероятностью приводит к опрокидыванию всех ячеек склона или значительной их части. Иначе говоря, чем шире граница, тем быстрее она смещается вбок. Таким образом, можно считать $V \sim w(t)$, что ведет преобразованию для скорости вида

$$
V \rightarrow b^{\chi} \cdot V
$$


Осреднение шума при огрублении должно проводиться вдоль границы, на которой он и действует. В силу ее фрактального характера, малые изменения $\varphi$ влекут большие изменения $h$. Поэтому можно приближенно считать, что именно величина $h$ характеризует пространственное положение границы, так что шум можно с приемлемой точностью представим в виде $\eta(t, h)$ с корреляцией $\left\langle\eta(t, h) \cdot \eta\left(t^{\prime}, h^{\prime}\right)\right\rangle \sim$ $\sim \delta\left(t-t^{\prime}\right) \cdot \delta\left(h-h^{\prime}\right)$. Соответственно, при перенормировке (32) шумовой член изменяется как

$$
\eta \rightarrow b^{-(z+x) / 2} \cdot \eta
$$

После перенормировки (32), выполненной с учетом формул (33) и (34), уравнение (31) принимает вид

$$
b^{\chi-z} \cdot \frac{\partial h}{\partial t}=b^{2 \chi-1} \cdot \frac{V}{r}\left|\frac{\partial h}{\partial \varphi}\right|+b^{-(\chi+z) / 2} \cdot \eta,
$$

который должен быть эквивалентен исходному, что требует выполнения скейлинговых соотношений

$$
\chi-z=2 \chi-1=-(\chi+z) / 2 \Leftrightarrow z=3 / 4, \quad \chi=1 / 4 .
$$

Таким образом, $r \sim t^{4 / 3}$ и $w \sim r^{1 / 4}$. Этот результат совпадает с формулами (22) и (23) при подстановке в них показателя (29) с тем единственным уточнением, что в модели роль времени из уравнения продвижения границы ожидаемо берет на себя кратность опрокидывания $m$. Двух полученных показателей связи $\gamma_{r m}=4 / 3$ и $\gamma_{w r}=1 / 4$ в сочетании со значениями (17) и (18) и тождеством (21) достаточно для почти полного решения модели. Единственной характеристикой, для которой показатели не рассчитываются напрямую, является длительность лавины. Однако они могут быть установлены благодаря совпадению периметра и активности.

Отдельным важным результатом проведенных расчетов является показатель связи

$$
\gamma_{C S}=5 / 8
$$

который пригодится далее при анализе модели БТВ. Для нахождения этого значения не требуется никаких показателей или соотношений, специфичных для рассматриваемой модели, а достаточно воспользоваться геометрически триальными показателем $\gamma_{S R}=2$ и соотношением $\gamma_{C R}=\gamma_{w r}+1$.

\section{4. Модель БТВ}

4.1. Предварительные замечания и общий анализ модели. Модель БТВ, которая в будущем году будет праздновать свое тридцатилетие, является исторически первой самоорганизованно-критической моделью. В ее изучение были вложены наибольшие усилия исследователей. Тем не менее, именно эта модель успешнее многих прочих сопротивлялась теоретическому решению. Поэтому рассказ о ней будет построен задом наперед, начинаясь с представления результатов компьютерного эксперимента и анонса значений показателей, которые будут рассчитаны далее.

Сложность модели БТВ связана, в первую очередь с тем, что к ней не вполне применим формализм конечно-размерного скейлинга, задаваемый формулами (1) и (2). В этой модели, как и в других, величины, описывающие происходящие события, 

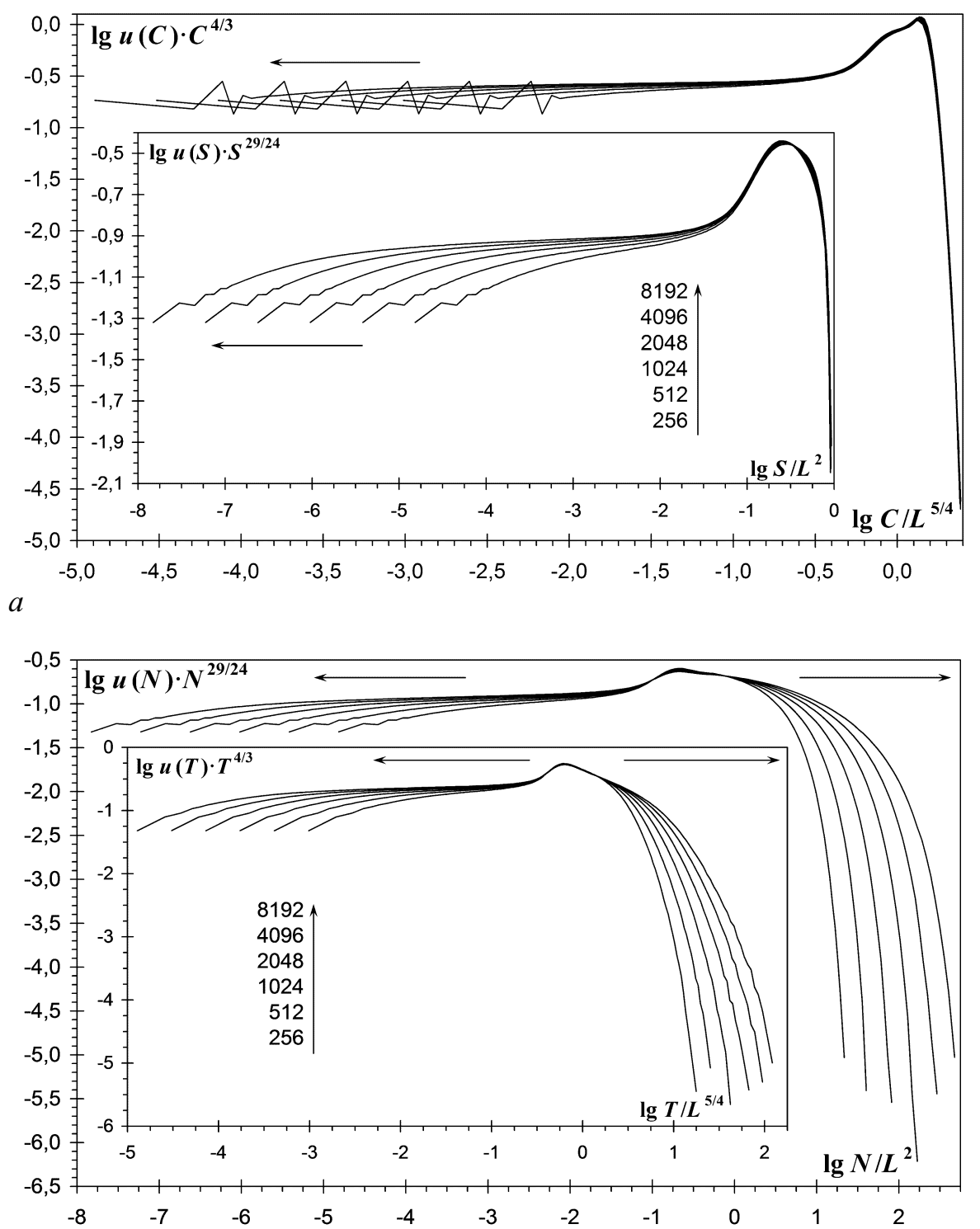

6

Рис. 7. Распределения лавин в модели БТВ: $a$ - скейлинговое поведение периметра и площади: при рассмотрении зависимости $u(x) \cdot x^{1+\alpha}$ от $x / L^{v}$ графики совпадают всюду, кроме области самых малых значений; $\sigma$ - нескейлинговое поведение размера и длительности - несмотря на совпадение показателей $\left(v_{N}=v_{S}=2, \alpha_{N}=\alpha_{S}=5 / 24\right.$ и $\left.v_{T}=v_{C}=5 / 4, \alpha_{T}=\alpha_{C}=1 / 3\right)$ отмасштабированные графики совпадают лишь в промежуточной асимптотике, а при $N / L^{v} \gg 1$ вновь расходятся

подчиняются степенному распределению в области промежуточной асимптотики. Ее правая граница, на которой плотность начинает отклоняться от степенного вида, как и в других моделях, дается формулой (2). Однако если для одних характеристик лавин скейлинговая функция стабилизируется при $L \rightarrow \infty$, то для других она оказывается существенно зависящей от размера системы.

Примеры обоих типов поведения приведены на рис. 7. Вероятность возникновения лавин, имеющих очень большие периметр или площадь, стремительно убывает по мере их увеличения, определяя вид скейлинговой функции. А вот размер и 
длительность лавин могут быть много больше значений, ограничивающих область промежуточной асимптотики. Причем в случае размера наличие таких - сверхкрупных - событий принципиальным образом сказываются на статистических свойствах модели.

В самом деле, формулы (17) и (18) сохраняют применимость для модели БТВ, так как на их выводе не сказываются минимальные отличия ее правил от правил модели Манны. Однако значение (18) несовместимо с формулой (3) и экспериментальным значением $v_{N}=2$, то есть средний размер лавины определяется именно теми событиями, которые не поместились в систему. Однако, не поместившись, они не прекращаются, переходя от «экстенсивного» развития, связанного с увеличением геометрических размеров, к «интенсивному», осваивающему ограниченную площадь. В результате на такие события приходится львиная доля опрокидываний.

Как видно из рис. 8, различия распределений площадей и размеров касаются лишь лавин, охвативших почти всю решетку. Однако за их счет существенно расширяется диапазон возможных размеров лавин.

В силу различия вида распределений совпадение показателей в паре размерплощадь (периметр-длительность) еще не означает близости этих величин, как это имело место для размера и площади в модели Федеров. Для умеренных событий, не чувствующих конечности размеров системы, можно считать, что $N \sim S(T \sim C)$, однако для сверхкрупных событий уже $N \gg S(T \gg C)$, хотя доля таких событий ничтожна.

В $55 \%+$ случаев вброс песчинки не нарушает устойчивость системы $(N=S=0)$, еще в $25 \%+$ случаев возникают лавины без повторных опрокидываний $(N=S>0)$ и лишь в $20 \%$ - случаев возникают повторные опрокидывания $(N>S)$. Распре-

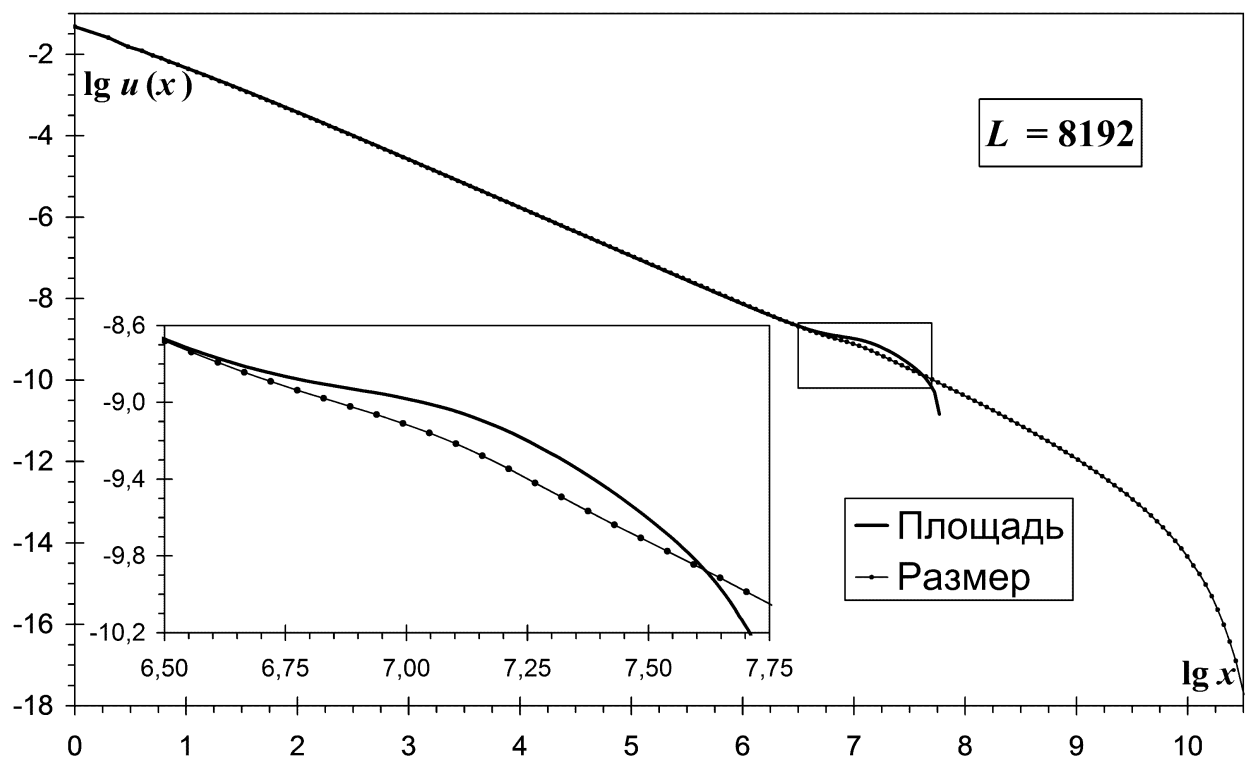

Рис. 8. Сравнение распределений лавин по размеру и площади в модели БТВ. Графики плотности для обеих характеристик совпадают почти всюду за исключением области самых больших значений. На врезке увеличен обведенный рамочкой фрагмент рисунка, где начинается расхождение графиков. За счет имеющегося здесь небольшого зазора между плотностями становятся возможны события с $N \gg L^{2}$ 
деления всех характеристик количественно преобладающих лавин без повторных опрокидываний подчиняются формулам (1) и (2) с теми же самыми скейлинговыми (но иными характеристическими) показателями. Это значит, что возможны крупные лавины, как с $N=S$, так и с $N \gg S$. Таким образом, условное распределение $u(N \mid S)$, равно как и другие условные распределения пар величин, описывающих происходящие события, нельзя считать узким. Вследствие этого показатель в формуле (4) оказывается зависящим от степени усреднения $q$, так что вопрос о том, можно ли связь между характеристиками лавины считать функциональной, начинает требовать экспериментальной проверки (впрочем, ничто не мешает рассматривать условное среднее как таковое).

Функциональной можно считать связь между площадью и периметром лавины, распределения которых демонстрируют скейлинговое поведение. Более того, показатель связи этих величин, оказывается одинаковым для моделей БТВ и Манны (причем это единственный совпадающий показатель связи для этих моделей), в чем позволяет убедиться рис. 9. Такой результат ожидаем, так как при получении значения (35) правила моделей учитывались максимально огрубленно, что не позволило проявиться различиям в их свойствах.

Области лавин в моделях БТВ и Манны при сходстве геометрии существенно различаются по структуре. Как уже было сказано, понятие слоя опрокидывания, будучи для модели Манны лишь общеописательным, для БТВ является вполне четким. В результате в ней все ячейки слоя, кроме граничных, опрокидываются в точности одно и то же число раз, отдавая и получая при этом строго равное количество песчинок. После лавины ячейки внутри слоя содержат столько же песчинок, сколько и до нее, а ячейки внутренней границы слоя - даже больше (внешней - меньше). В таких условиях лавина может прекратиться, только если внутренняя и внешняя

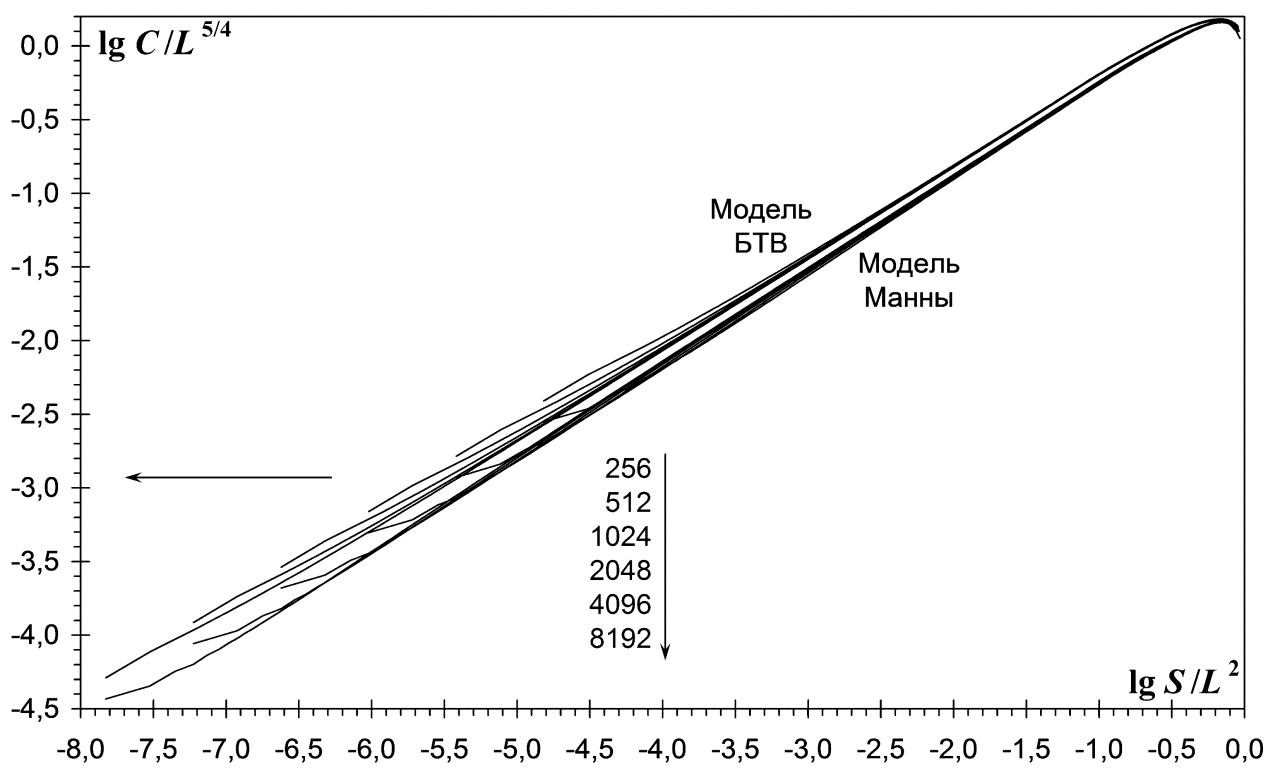

Рис. 9. Связь периметра и площади для изотропных консервативных моделей. Для обеих моделей рассматриваемые величины имеют одинаковые скейлинговые показатели $v_{S}=2, v_{C}=5 / 4$. Поэтому в силу соотношения (5) и показатель связи для них один и тот же 


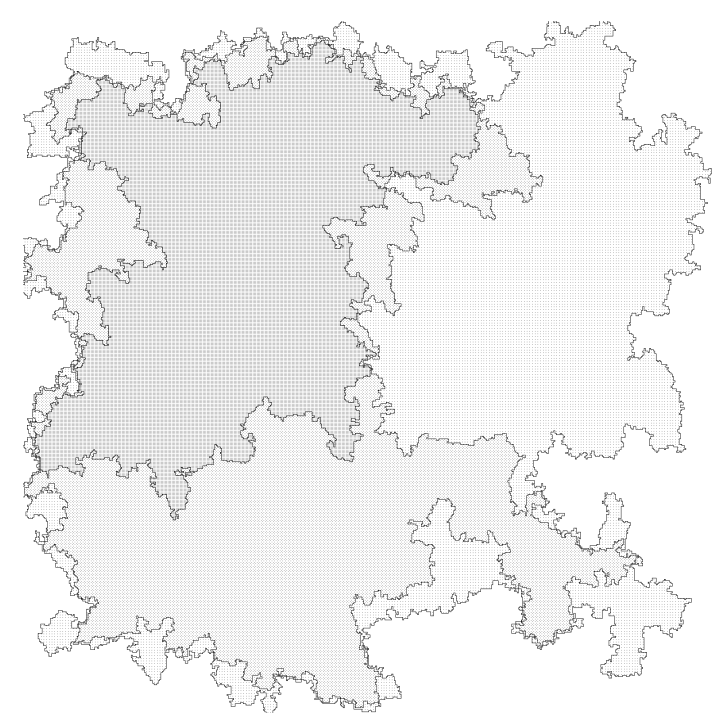

Рис. 10. Пример слоев опрокидывания для крупной лавины в модели БТВ. Точка вброса (в середине решетки) лежит на границе области максимальной кратности опрокидывания. Толщина окружающих ее слоев в некоторых местах составляет всего одну ячейку. Размер решетки $L=512$. Лавина затронула $S=196990$ ячеек, в ее ходе произошло $N=380107$ опрокидываний и за край выпало $F=45$ песчинок. Максимальная кратность опрокидывания (она же - число волн) $m=3$

границы слоя где-то совпадут, из-за чего ячейки, принадлежащие одновременно к обеим границам, все-таки вернут состояние, которое имели до лавины, а с ним - и устойчивость. В модели Манны альтернативой были флуктуации при передаче песчинок, но в модели БТВ такой возможности нет, в результате чего каждый слой ее лавин в каких-то местах истончается до единичной ширины, как показано на рис. 10. Следствием этого является невозможность роста ширины слоев с их номером, что в силу формулы (23) дает тривиальное значение $\gamma=1$. Иными словами, формула (22) упрощается до записи

$$
r_{k} \sim k
$$

коэффициент пропорциональности в которой, однако, сам по себе является случайной величиной, чем и обеспечивается возможность лавин как с единичной, так и с очень большой кратностью опрокидывания при одном и том же радиусе.

Наряду с пространственной декомпозицией области лавины посредством рассмотрения слоев опрокидывания возможна и временна́я декомпозиция процесса ее развития посредством рассмотрения волн опрокидывания [18-20], под которыми понимается такой порядок обработки неустойчивых ячеек, при котором опрокидывание ячейки вброса осуществляется только тогда, когда нет других неустойчивых ячеек. При этом каждое ее опрокидывание начинает новую волну, которая, распространяясь от точки вброса вовне, создает новые слои. Соответственно границы распространения волн совпадают с границами слоев, возникающих по завершении лавины, хотя вложенность волн не обязана совпадать со слоями, поскольку во время одной лавины иногда могут возникать такие пары волн, ни одна из которых не вкладывается в другую.

Как показывает моделирование, в подавляющем большинстве случаев последующие волны не пересекают границы предыдущих. Вместе с тем, в среднем размер 


\begin{tabular}{|c|c|c|c|c|c|c|c|c|c|c|c|c|c|c|c|c|}
\hline & 2 & 2 & 2 & 3 & 0 & & 33 & 33 & 3 & 0 & & 3 & 3 & & & \\
\hline & 3 & 3 & 3 & 1 & 3 & & $\overline{02}$ & 1 & 3 & 3 & & 0 & 3 & & & 3 \\
\hline & 0 & 3 & 4 & 3 & 1 & 3 & 32 & 4 & 1 & 2 & 3 & 4 & 0 & & & 2 \\
\hline & 3 & 3 & 3 & 3 & 2 & 3 & 03 & 3 & 1 & 3 & 3 & 1 & 1 & & & $\mid 3$ \\
\hline & 1 & 3 & 3 & 1 & 3 & 3 & 31 & 1 & 3 & 3 & 3 & 3 & 2 & & & 3 \\
\hline & & & 2 & & 0 & 0 & & & 3 & 0 & 0 & & & & & 0 \\
\hline
\end{tabular}

Рис. 11. Пример прорыва границы волны опрокидывания. Слева - состояние системы перед началом первой волны. Тонкой рамочкой обведена точка вброса - единственная неустойчивая ячейка. Посередине - состояние системы перед началом второй волны. Граница первой волны показана толстой линией. Ячейки, примыкающие к ней изнутри, потеряли песчинки, а примыкающие снаружи - приобрели. Справа - момент развития второй волны, когда она выходит за пределы первой. После этого опрокидывания начинают распространяться вдоль ее внешней границы

следующей волны оказывается больше размера предыдущей (за исключением волн, соизмеримых с площадью решетки, которым уже просто некуда дальше расти) [21]. Такое поведение связано с уже упомянутым возникновением на границе слоя двух рядов ячеек, в первом из которых количество песчинок уменьшилось, а во втором увеличилось. Внешняя граница слоя, состоящая из обедненных ячеек, представляет существенное препятствие для распространения очередной волны опрокидывания (50\% вероятности того, что лавина выйдет за пределы первой волны, соответствует площадь последней примерно в 500 ячеек). Однако если прорваться на следующий слой все же удается (простейший пример того, как это происходит, показан на рис. 11), то далее опрокидывания с высокой вероятностью подхватываются его внутренней границей, состоящей из обогащенных ячеек, легко распространяясь сначала вдоль нее, а затем - и вовне.

Такое поведение, сопряженное с ожиданием удачных условий для прорыва через обедненные ячейки и последующего обхода обогащенных, не обязательно требует полностью сформированной границы. Оно возможно и на ее незамкнутых фрагментах, возникающих в тех местах, которые потом могут оказаться внутри слоев. Как бы то ни было, в силу фрактального характера границ, движение вдоль них требует большого времени, чем и объясняется совпадение показателей для периметра и длительности. А редкостью события прорыва границы объясняется совпадение показателей для площади и размера, ведущее к малой кратности опрокидывания. Поэтому, несмотря на то, что для модели БТВ ни средняя, ни максимальная кратности опрокидывания не демонстрируют скейлингового поведения, для них можно ожидать выполнения формулы (20), что подтверждает рис. 12. Таким образом, эта формула является более общей, чем единичный характеристический показатель (19), на основе которого она была выведена для модели Манны.

В отличие от лавин, волны опрокидывания описываются скейлинговыми распределениями, для которых был строго получен ряд важных теоретических результатов. В частности, в работах $[12,20,22]$ определен вид распределения волн по размеру и выведена формула

$$
\langle m\rangle \sim \log L
$$




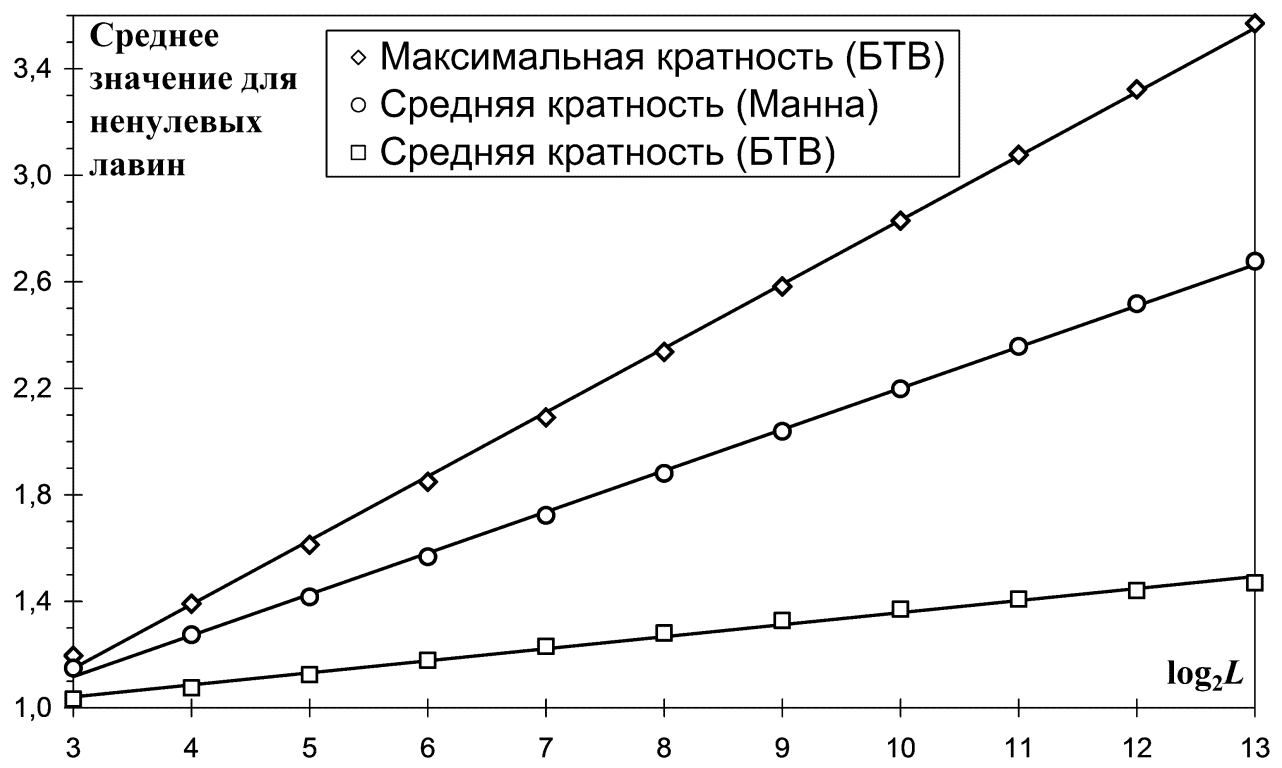

Рис. 12. Кратность опрокидывания в моделях Манны и БТВ. Прямыми линиями показаны аппроксимации экспериментальных зависимостей. Для $\left\langle m_{\text {Бтв }}\right\rangle$ и $\left\langle M_{\text {Манна }}\right\rangle$ линейные апроксимации практически идеальны, тогда как $\left\langle M_{\text {Бтв }}\right\rangle$, видимо, растет чуть медленнее, чем логарифм размеров решетки

являющаяся аналогом формулы (20). А в работах $[18,23]$ показано, что периметр волн пропорционален их длительности, а та связана с их радиусом формулой $t \sim r^{5 / 4}$, что в совокупности воспроизводит значение (35). Поэтому в адекватности общефизических соображений, высказанных до настоящего момента, можно не сомневаться, тогда как дальнейшие рассуждения подкрепляются лишь компьютерным экспериментом и потому не столь надежны.

4.2. Решение модели и сопоставление с моделью Манны. Возникновение повторных волн опрокидывания является в значительной мере случайным процессом. Это означает, что хотя параметры условного распределения $u(m \mid x)$, где $x-$ некоторая характеристика лавины, и зависят от $x$, само распределение не является узким. Поэтому к величине $m$ неприменим весь тот формализм, который использовался для решения прочих самоорганизованно-критических моделей.

Вместе с тем, как показывает моделирование, условное среднее $m(x)$, изменяется как некоторая степень $x$ (а вот обратное уже неверно - зависимость $x(m)$ не является степенной функцией). Опираясь на этот экспериментальный факт, с помощью формулы (37) и записи

$$
\langle m\rangle=\int^{L^{v_{x}}} m(x) \cdot x^{-\left(1+\alpha_{x}\right)} d x
$$

приходим к тождеству

$$
\gamma_{m x}=\alpha_{x}
$$

аналогичному тождеству (21) для модели Манны.

Если рассматривать инициацию волн опрокидывания с течением времени как несимметричное случайное блуждание, то простейшее предположение о длинах скачка из формулы (11) будет иметь вид $\delta_{-}=$const, $\delta_{+} \sim 1 / m$, так как новой волне 
нужно «втиснуться» в пространство лавины, поделенное между уже возникшими волнами. Таким образом, в формуле (9) имеем $\tau=0, \xi=-1$ и соотношение (10) дает $\gamma_{m T}=1 / 3$. Непосредственная проверка этого значения по результатам компьютерного моделирования проблематична, поскольку любой алгоритм обеспечивает либо параллельную обработку всех потерявших устойчивость ячеек, либо блокировку ячейки вброса для описания развития волн, но не то и другое одновременно. Однако, исходя из совпадения показателей для длительности лавины и ее периметра, можно ожидать соотношения $\gamma_{m C}=1 / 3$, в правдоподобии которого позволяет удостовериться рис. 13.

Тождество (38) дает характеристические показатели для длительности и периметра $\alpha_{T}=\alpha_{C}=1 / 3$, на основе которых находится универсальный показатель $\varepsilon=5 / 12$ и остальные характеристические показатели $\alpha_{N}=\alpha_{S}=5 / 24$, которые ранее были использованы для построения графиков на рис. 7.

Несмотря на удовлетворительное совпадение полученных значений с результатами эксперимента, к проведенным расчетам следует относиться с определенной осторожностью, учитывая, что использованная методика не была апробирована на другом материале и опирается на интуитивно неочевидные экспериментальные данные.

В завершение рассмотрения модели БТВ вернемся к природе ее отличий от модели Манны, попытавшись соотнести развитые для них системы понятий.

Если распространить на модель Манны представление о волнах опрокидывания (следуя не буквальному определению, а сохраняя физический смысл), они в ней будут вести себя не так, как в модели БТВ. Здесь границы слоев размыты и на них нет сплошных рядов из обедненных и обогащенных ячеек. Поэтому границы не представляют собой принципиального препятствия для волн опрокидывания (имен-

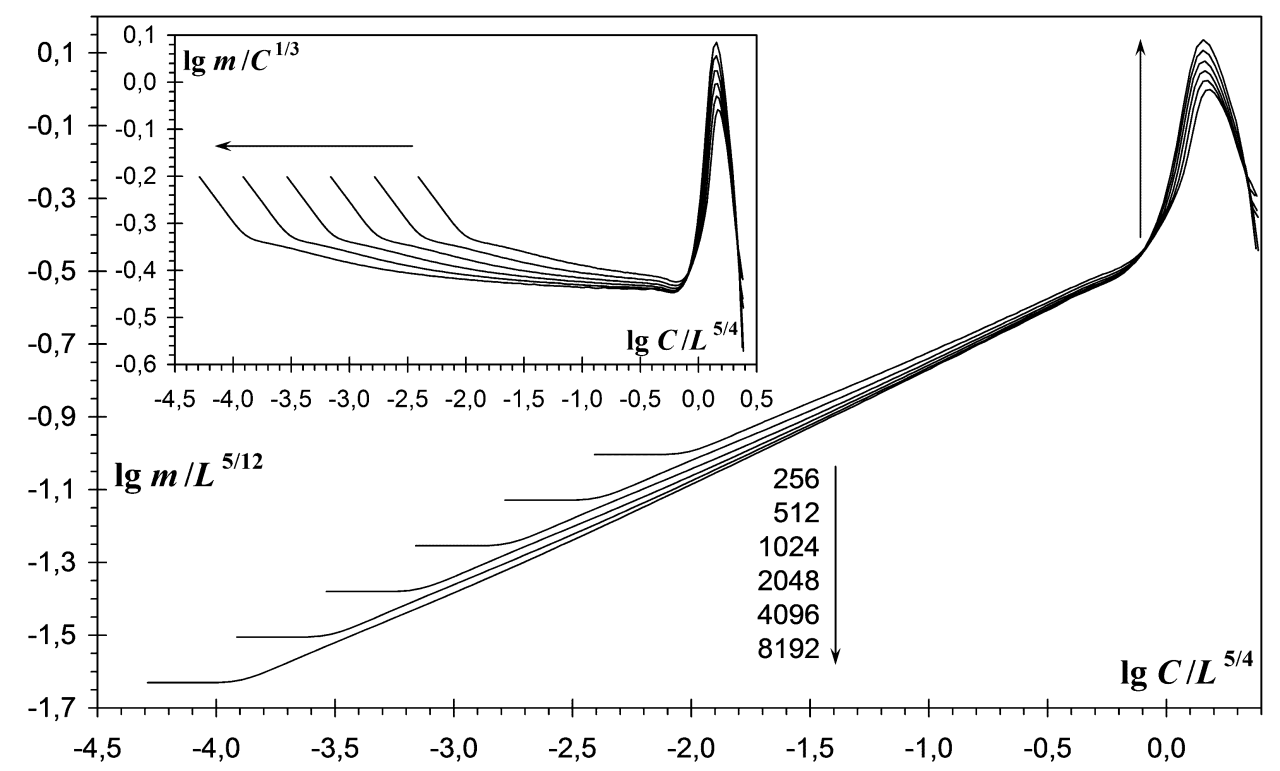

Рис. 13. Связь числа волн и периметра области лавины в модели БТВ. После нормировки переменных на $L^{v}$ графики, полученные при разных размерах решетки, совпадают. Врезка демонстрирует уменьшение отклонения от зависимости между рассматриваемыми величинами по мере роста размера системы 

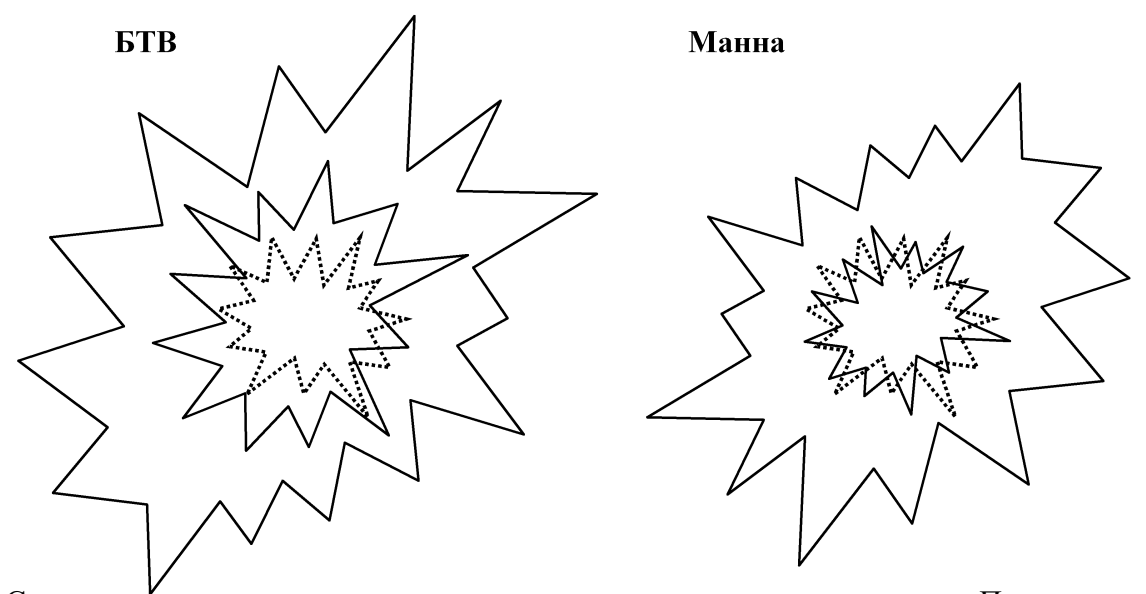

Рис. 14. Схематичное представление изменения границ слоев опрокидывания. Пунктиром показана граница единственного слоя после прохождения первой волны опрокидывания, сплошными линиями границы между слоями после прохождения второй волны. В модели Манны волна просто добавляет к слою новый, тогда как в модели БТВ она еще и смещает границу предыдущего слоя

но этим объясняется то, что заполнение ячеек в модели Манны $\langle z\rangle \approx 2.056$ ниже, чем в модели БТВ $\langle z\rangle \approx 2.125$ (в пределе $L \rightarrow \infty$ ). Однако поскольку нет и никаких дополнительных преимуществ в распространении опрокидывания вдоль границы, после прохождения очередной волны предыдущий слой сохраняет свои размеры. Волна просто увеличивает кратность опрокидывания внутри каждого пройденного ею слоя без систематического смещения их границ. Иными словами, при прохождении волн в модели Манны границы слоев смещаются лишь случайным образом, а в модели БТВ они испытывают, кроме того, и систематический сдвиг, направленный вовне (см. рис. 14). Таким образом, обнаруживается различие между моделями БТВ и Манны на уровне симметрий. Только это не статические симметрии правил, которые у моделей совпадают, а динамические симметрии тех самоорганизационных процессов, которые обеспечивают пребывании систем в критическом состоянии.

С другой стороны, если попытаться распространить на модель БТВ представление об активных частицах, то вместо выражения $a_{k} \sim r_{k}^{1 / 2}$, которое определение (26) давало для модели Манны, из формул (35) и (36) получается $a_{k} \sim r_{k}^{1 / 4}$ (следует подчеркнуть, что формула (25) неприменима к модели БТВ в силу принципиально иной структуры слоев опрокидывания, так что пользоваться следует именно общей формулой (35)). Таким образом, локальная активность на одном и том же расстоянии от точки вброса в этих моделях связана формальной зависимостью $a_{\text {Манна }} \sim a_{\text {Бтв }}^{2}$. Модели Манны требуется принципиально больше активных частиц, чем модели БТВ, так как в первой модели они двигаются через слои с практически неподвижными границами, тогда как во второй - они могут перемещаться и вместе с подвижными границами слоев.

\section{Выводы}

Для большинства самоорганизованно-критических моделей непосредственное определение на основе их правил показателей распределений, описывающих происходящие в этих моделях события, является нетривиальной задачей. Важным под- 
спорьем служит формулировка моделей промежуточного уровня, с одной стороны, описывающих коллективную динамику для исходных модели, а с другой - допускающих исследование с помощью сравнительно простого математического аппарата.

Этот подход, опирающийся на физическую интуицию и компьютерный эксперимент, был применен к 5 моделям кучи песка. В таблице собраны полученные в ходе их решения скейлинговые и универсальные показатели. Эти значения позволяют рассчитать все остальные показатели: характеристические - из соотношения (7), показатели связи - из соотношения (5) и вторые скейлинговые показатели - из соотношения (3). Однако для ключевых выводов достаточно данных, представленных в таблице.

Важнейшей характеристикой моТаблица дели является универсальный показатель $\varepsilon$, зависимость которого от ее особенностей представляется наиболее интересной. Чем он больше, тем легче крупным лавинами помещаться в систему конечного размера. Введение избранного направления может увеличить универсальный показатель

\begin{tabular}{|c|c|c|c|c|c|}
\hline Модель & БТВ & ДР & Федеров & ПСВ & Манны \\
\hline$v_{N}$ & $2^{*}$ & $1 \frac{1}{2}$ & $1 \frac{2}{3}$ & $1 \frac{3}{4}$ & $2 \frac{3}{4}$ \\
\hline$v_{N}$ & 2 & $1 \frac{1}{2}$ & $1 \frac{2}{3}$ & $1 \frac{1}{2}$ & 2 \\
\hline$v_{r}$ & $1 \frac{1}{4}^{*}$ & 1 & 1 & 1 & $1 \frac{1}{2}$ \\
\hline$v_{C}$ & $1 \frac{1}{4}$ & 1 & 1 & $1 \frac{1}{4}$ & $1 \frac{1}{4}$ \\
\hline$\varepsilon$ & $\frac{5}{12}$ & $\frac{1}{2}$ & $\frac{2}{3}$ & $\frac{3}{4}$ & $\frac{3}{4}$ \\
\hline
\end{tabular}

${ }^{*}$ Нескейлинговое поведение $\left(\varepsilon_{\text {Бтв }}<\varepsilon_{\text {дР }}\right)$, но может и не увеличить $\left(\varepsilon_{\text {Манны }}=\varepsilon_{\text {ПСВ }}\right)$. Очевидно, его увеличивает диссипация $\left(\varepsilon_{\text {Бтв }}<\varepsilon_{\text {Федеров }}\right)$. Однако сильнее всего на нем сказывается стохастичность $\left(\varepsilon_{\text {БТВ }}<\varepsilon_{\text {Манны и }} \varepsilon_{\text {ДР }}<\varepsilon_{\text {ПСВ }}\right)$.

Стохастичность проявляет себя еще и неравенствами $v_{N}>v_{S}$ и $v_{C}>v_{T}$, тогда как для всех рассмотренных детерминированных моделей $v_{N}=v_{S}$ и $v_{C}=v_{T}$. Иначе говоря, в стохастических моделях лавины имеют больше разнообразных осмысленных характеристик, чем в детерминированных. По этой причине для решения стохастических моделей приходилось рассматривать два процесса случайного блуждания, а для детерминированных хватало и одного.

Важнейшим следствием детерминированности правил оказывается возникновение динамических симметрий. В модели ДР вся эволюция лавин сосредотачивается на их границе, которая тем самым становится выделенной частью области лавины, тогда как в модели ПСВ существенные события происходят и в объеме. В модели Федеров имеет место спонтанная анизотропия, направляющая активность от краев решетки к ее середине, несмотря на изотропию правил. Наконец, в модели БТВ при смещении границ слоев проходящими волнами нарушается симметрия между направлениями наружу и вовнутрь лавины, тогда как в модели Манны эти направления равноправны.

Работа выполнена при поддержке РФФИ (проекты 14-01-00773 и 16-01-00342).

\section{Библиографический список}

1. Бак П. Как работает природа: Теория самоорганизованной критичности / Пер. с англ. Изд.стереотип. / Синергетика: от прошлого к будущему. № 66. M.: URSS, 2015. $276 \mathrm{c}$. 
2. Dhar D., Ramaswamy R. Exactly solved model of self-organized critical phenomena // Phys. Rev. Lett. 1989. Vol. 63, N16. Pp. 1659-1662.

3. Pastor-Satorras R., Vespignani A. Universality classes in directed sandpile models // J. Phys. A. 2000. Vol. 33, N3. Pp. L33-L39.

4. Feder H.J.S., Feder J. Self-organized criticality in a stick-slip process // Phys. Rev. Lett. 1991. Vol. 66, N20. Pp. 2669-2672.

5. Manna S.S. Two-state model of self-organized criticality // J. Phys. A. 1991. Vol. 24, N7. Pp. L363-L639.

6. Bak P., Tang C., Wiesenfeld K. Self-organized criticality: An explanation of $1 / \mathrm{f}$-noise // Phys. Rev. Lett. 1987. Vol. 59, N4. Pp. 381-384.

7. Bak P., Tang C., Wiesenfeld K. Self-organized criticality // Phys. Rev. A. 1988. Vol. 38, N1. Pp. 364-374.

8. Dhar D., Pruessner G., Expert P., Christensen K., Zachariou N. Directed Abelian sandpile with multiple downward neighbors // Phys. Rev. E. 2016. Vol. 93. 042107.

9. Paczuski M., Bassler K.E. Theoretical results for sandpile models of SOC with multiple topplings // Phys. Rev. E. 2000. Vol. 62, Iss. 4. Pp. 5347-5352.

10. Kloster M., Maslov S., Tang C. Exact solution of stochastic directed sandpile model // Phys. Rev. E. 2001. Vol. 63. 026111.

11. Hu C.K., Ivashkevich E.V., Lin C.Y., Priezzhev V.B. Inversion symmetry and exact critical exponents of dissipating waves in the sandpile model // Phys. Rev. Lett. 2000. Vol. 85, N19. Pp. 4048-4051.

12. Ktitarev D.V., Lübeck S., Grassberger P., Priezzhev V.B. Scaling of waves in the Bak-Tang-Wiesenfeld sandpile model // Phys. Rev. E. 2000. Vol. 61, N1. Pp. 8192.

13. Hughes D., Paczuski M. Large scale structures, symmetry, and universality in sandpiles // Phys. Rev. Lett. 2002. Vol. 88. 054302.

14. Подлазов А.В. Двумерные самоорганизованно-критические модели типа кучи песка с анизотропной динамикой распространения активности// Изв. вузов. ПНД. 2012. Т. 20, N6. С. 25-46.

15. Малинеикий Г.Г., Подлазов А.В. Сравнение двумерных изотропных консервативных самоорганизованно-критических моделей типа кучи песка // Инженерный журнал: Наука и инновации. 2012. Т. 4, № 4.167.

16. Подлазов А.В. Решение двумерной самоорганизованно-критической модели Манны // Изв. вузов. ПНД. 2013. Т. 21, № 6. С. 69-87.

17. Подлазов А.В. Двумерная самоорганизованно-критическая модель Манны для песчаных сред // Мат. модел. и числ. мет. 2014, № 3(3). С. 89-110.

18. Ivashkevich E.V., Ktitarev D.V., Priezzhev V.B. Critical exponents for boundary avalanches in a 2D Abelian sandpile // J. Phys. A: Math. Gen. 1994. Vol. 27, N16. Pp. L585-L590.

19. Dhar D., Manna S.S. Inverse avalanches in the Abelian sandpile model // Phys. Rev. E. 1994. Vol. 49, N4. Pp. 2684-2687.

20. Ivashkevich E.V., Ktitarev D.V., Priezzhev V.B. Waves of topplings in an Abelian sandpile // Physica A. 1994. Vol. 209, N3-4. Pp. 347-360. 
21. Paczuski M., Boettcher S. Avalanches and waves in the Abelian sandpile model // Phys. Rev. E. 1997. Vol. 56, N4. Pp. R3745-R3748.

22. Priezzhev V.B., Ktitarev D.V., Ivashkevich E.V. Formation of avalanches and critical exponents in Abelian sandpile model // Phys. Rev. Lett. 1996. Vol. 76, N12. Pp. 2093-2096.

23. Majumdar S.N., Dhar D. Equivalence between the Abelian sandpile model and the $q \rightarrow 0$ limit of the Potts model // Physica A. 1992. Vol. 185, N1-4. Pp. 129-145.

Поступила в редакциию

01.09 .2016

\title{
CLASSICAL TWO-DIMENSIONAL SANDPILE MODELS
}

\author{
A. V. Podlazov \\ Russian Academy of Sciences Keldysh Institute of Applied Mathematics \\ Miusskaya sq., 4, Moscow, 125047, Russia \\ E-mail: tiger-cub@mail.ru
}

I consider sandpile models being open nonlinear systems demonstrating the phenomenon of avalanche-like response to a single disturbance of steady state. I study thoroughly the five most known variants of the two-dimensional rules referred as the models of Dhar-Ramaswamy, Pastor-Satorras-Vespignani, Feder-Feder, Manna and Bak-Tang-Wiesenfeld. The analytical solutions obtained in various ways are known for the first four models and the reasons preventing the construction of a solution are known for the fifth one. The generalization of these results allows to develop a common approach to the theoretical study of self-organized critical phenomena.

Self-organization into the critical state gives rise to the scale-invariant properties. Theirs statistical description can not be generally obtained on the basis of the models' rules. Intermediate level models mediates between the microlevel of the elements local behavior and the macrolevel of the entire system behavior. The rules of these models are not derived from the rules of original models, but are formulated on the ground of physical intuition, computer simulation results and common understanding of the dynamic processes that hold the system near the critical point.

The collective dynamics of all of the models is reduced at the intermediate level to the processes familiar to mathematical physics, the first of them are asymmetric random walks. On this basis, I propose uniform methods of solution of models. The BTW model is solved for the first time.

All the critical indices are analytically calculated for the models considered. The influence of the rules features of models on their common properties is analysed on theses ground.

The most important for the rules is the aspect whether they are stochastic or deterministic. The former increases the number of avalanche characteristics with different properties, and the later helps large avalanches fit into a finite-size system and results in the system as a whole obtaining dynamic symmetries absent at the level of elements behaviour rules.

Keywords: Self-organized criticality, sandpile models, scale invariance, power laws, finite-size scaling, meso-level models, random walks, spontaneous anisotropy, dynamical symmetries.

DOI: $10.18500 / 0869-6632-2016-24-4-39-70$

Paper reference: Podlazov A.V. Classical two-dimensional sandpile models // Izvestiya VUZ. Applied Nonlinear Dynamics. 2016. Vol. 24, N4. P. 39-70. 


\section{References}

1. Bak P. How nature works: The science of self-organized criticality. Springer-Verlag, New York, Inc. 1996.

2. Dhar D., Ramaswamy R. Exactly solved model of self-organized critical phenomena // Phys. Rev. Lett. 1989. Vol. 63, N16. Pp. 1659-1662.

3. Pastor-Satorras R., Vespignani A. Universality classes in directed sandpile models // J. Phys. A. 2000. Vol. 33, N3. Pp. L33-L39.

4. Feder H.J.S., Feder J. Self-organized criticality in a stick-slip process // Phys. Rev. Lett, 1991. Vol. 66, N20, pp. 2669-2672.

5. Manna S.S. Two-state model of self-organized criticality // J. Phys. A. 1991. Vol. 24, N7. Pp. L363-L639.

6. Bak P., Tang C., Wiesenfeld K. Self-organized criticality: An explanation of $1 / \mathrm{f}-$ noise // Phys. Rev. Lett. 1987. Vol .59, N4. Pp. 381-384.

7. Bak P., Tang C., Wiesenfeld K. Self-organized criticality // Phys. Rev. A. 1988. Vol. 38, N1. Pp. 364-374.

8. Dhar D., Pruessner G., Expert P., Christensen K., Zachariou N. Directed Abelian sandpile with multiple downward neighbors // Phys. Rev. E. 2016. Vol. 93. 042107.

9. Paczuski M., Bassler K.E. Theoretical results for sandpile models of SOC with multiple topplings // Phys. Rev. E. 2000. Vol. 62, N4. Pp. 5347-5352.

10. Kloster M., Maslov S., Tang C. Exact solution of stochastic directed sandpile model // Phys. Rev. E. 2001. Vol. 63. 026111.

11. Hu C.K., Ivashkevich E.V., Lin C.Y., Priezzhev V.B. Inversion symmetry and exact critical exponents of dissipating waves in the sandpile model // Phys. Rev. Lett. 2000. Vol. 85, N19. Pp. 4048-4051.

12. Ktitarev D.V., Lübeck S., Grassberger P., Priezzhev V.B. Scaling of waves in the Bak-Tang-Wiesenfeld sandpile model // Phys. Rev. E. 2000. Vol. 61, N1. Pp. 81-92.

13. Hughes D., Paczuski M. Large scale structures, symmetry, and universality in sandpiles // Phys. Rev. Lett. 2002. Vol. 88. 054302.

14. Podlazov A.V. Two-dimensional self-organized critical sandpile models with anisotropic dynamics of the activity propagation// Izvestiya VUZ. AND. 2012. Vol. 20, N6. Pp. 25-46.

15. Malinetskiy G.G., Podlazov A.V. Comparison of two-dimensional isotropic conservative self-organized critical sandpile models // Engineering Journal: Science and Innovation. 2012. Vol. 4, N4. 167.

16. Podlazov A.V. Solution of two-dimensional self-organized critical Manna model // Izvestiya VUZ. AND. 2013. Vol. 21, N6. Pp. 69-87.

17. Podlazov A.V. Two-dimensional self-organized critical Manna model // Mathematical modeling and computational methods. 2014. Vol. 3, N3. Pp. 89-110.

18. Ivashkevich E.V., Ktitarev D.V., Priezzhev V.B. Critical exponents for boundary avalanches in a 2D Abelian sandpile // J. Phys. A: Math. Gen. 1994. Vol. 27, N16. Pp. L585-L590. 
19. Dhar D., Manna S.S. Inverse avalanches in the Abelian sandpile model // Phys. Rev. E. 1994. Vol. 49, N4. Pp. 2684-2687.

20. Ivashkevich E.V., Ktitarev D.V., Priezzhev V.B. Waves of topplings in an Abelian sandpile // Physica A. 1994. Vol. 209, N3-4. Pp. 347-360.

21. Paczuski M., Boettcher S. Avalanches and waves in the Abelian sandpile model // Phys. Rev. E. 1997. Vol. 56, N4. Pp. R3745-R3748.

22. Priezzhev V.B., Ktitarev D.V., Ivashkevich E.V. Formation of avalanches and critical exponents in Abelian sandpile model // Phys. Rev. Lett. 1996. Vol. 76, N12. Pp. 2093-2096.

23. Majumdar S.N., Dhar D. Equivalence between the Abelian sandpile model and the $q \rightarrow 0$ limit of the Potts model // Physica A. 1992. Vol. 185, N1-4. Pp. 129-145.

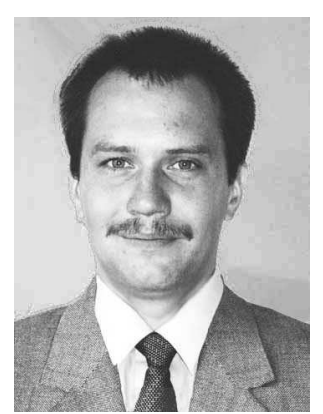

Подлазов Андрей Викторович - родился в Москве (1973), окончил Московский физико-технический институт (1996). После окончания МФТИ работает в Институте прикладной математики им. М.В. Келдыша РАН старшим научным сотрудником. Защитил диссертацию на соискание ученой степени кандидата физико-математических наук в ИПМ им. М.В. Келдыша РАН (2001) в области теории самоорганизованной критичности и теории масштабно-инвариантных процессов. Автор монографий «Управление риском. Риск, устойчивое развитие, синергетика» [М.: Наука, 2000. 432 с.] (в соавторстве с В.А. Владимировым, Ю.Л. Воробьевым, Г.Г. Малинецким и др.) и «Нелинейная динамика: Подходы, результаты, надежды» [Изд.3/ Синергетика: от прошлого к будущему. М.: ЛИБРОКОМ, 2011. 280 с.] (в соавторстве с Г.Г. Малинецким и А.Б. Потаповым). Опубликовал 60 научных статей по направлениям, указанным выше, а также по демографии, математической истории и вопросам развития системы образования.

125047 Москва, Миусская пл., 4

ИПМ им. М.В. Келдыша РАН

E-mail: Tiger@Keldysh.ru 